\title{
Patterning metallic electrodeposits with magnet arrays
}

\author{
Peter Dunne* and J. M. D. Coey \\ School of Physics and CRANN, Trinity College, Dublin 2, Ireland
}

(Received 22 December 2011; published 12 June 2012)

\begin{abstract}
The influence of a pattern of a magnetic field on the structure of metal deposits at the cathode of a small electrochemical cell is investigated for cobalt, nickel, copper, and zinc. The different magnetic properties of the ions in their oxidized and reduced states, together with the influence on the patterned electrodeposits of variables, including the structure of the array of small magnets used to generate the field pattern, applied magnetic field, ion concentration, cell orientation, and deposition time lead to an understanding of the physical processes involved. The results for direct deposits from paramagnetic cations such as $\mathrm{Cu}^{2+}$ when convection is minimized are largely explained in terms of magnetic pressure, which modifies the thickness of the diffusion layer that governs mass transport. Patterning is governed by the susceptibility of the electroactive species relative to the nonelectroactive background. No patterning is observed until the diffusion layer begins to form, as it requires orthogonal concentration and magnetic field gradients. An inverse effect, whereby deposits are structured in complementary patterns, such as antidot arrays, is observed when a strongly paramagnetic but nonelectroactive cation such as $\mathrm{Dy}^{3+}$ is present in the electrolyte, together with an electroactive cation such as $\mathrm{Cu}^{2+}$ or $\mathrm{Zn}^{2}$. Inverse patterning is related to magnetically induced convection produced by the inhomogeneous magnetic field. Blocking of sites in the double layer by the rare-earth ions may also be involved. The inverse deposits are concentrated in regions where the magnitude of the field is lowest; they can also be produced directly by superposing a uniform magnetic field on that of the magnet array.
\end{abstract}

DOI: 10.1103/PhysRevB.85.224411

PACS number(s): 47.35.Tv, 47.65.Cb, 82.45.Qr, 82.47.Wx

\section{INTRODUCTION}

The influence of magnetic fields on electrodeposition of metals has been investigated sporadically for more than 25 years. ${ }^{1-16}$ Much of the early work was concerned with the influence of uniform fields, applied parallel to the cathode in order to maximize the Lorentz force

$$
\boldsymbol{F}_{\mathrm{L}}=\boldsymbol{j} \times \boldsymbol{B},
$$

where $\boldsymbol{j}$ is the current density in the cell and $\boldsymbol{B}$ is the applied magnetic flux density. The magnetically induced convection produced by $\boldsymbol{F}_{\mathrm{L}}$, known as the magnetohydrodynamic (MHD) effect, may be calculated by solving the convection-diffusion equation in conjunction with the Navier-Stokes equation with Eq. (1) as a driving force. The MHD effect, and its analog on a small length scale, the micro-MHD effect ${ }^{17}$ are responsible for many of the influences that a magnetic field can have in electrochemistry, including the rate of electrodeposition of metals, ${ }^{3,5,7}$ deposit morphology,${ }^{8}$ hydrogen production,,${ }^{10,11}$ corrosion, ${ }^{12-16}$ and rest potential. ${ }^{18}$ The hydrodynamic conditions in any experiment are characterized by a set of dimensionless numbers whose significance in a laboratory electrochemical cell is summarized in Table I.

There is growing interest in the influence of nonuniform magnetic fields on electrochemical reactions, ranging from confinement of organic species at disc microelectrodes ${ }^{19}$ to remapping of MHD flows ${ }^{20}$ and spatially correlated suppression of corrosion of iron wires. ${ }^{21}$ Previous work on magnetic structuring of electrodeposits includes reports on the preferential deposition of $\mathrm{Cu}$ in the vicinity of the field of a magnet ${ }^{22}$ and electrodeposit patterning by magnetized iron wires. ${ }^{23}$ A preliminary account of some of the present experiments on normal and inverse deposition was published recently, ${ }^{24}$ and there has been a report on inverse deposition of bismuth in the presence of $\mathrm{Mn}^{2+} .25$
Electrodeposition is often carried out under steady-state conditions, where the current is mass transport limited. In these circumstances, a profile with a uniform concentration gradient of the electroactive species is established in the vicinity of the cathode, and the limiting diffusion current

$$
j_{o}=-\mathrm{nF} D \nabla c
$$

is a constant. Convection, whether natural, forced, or driven by the Lorentz force, determines the thickness $\delta$ of the diffusion layer, a region near the cathode about $100 \mu \mathrm{m}$ thick, where there is a uniform concentration gradient $\nabla c=c_{0} / \delta$ of the electroactive species, whose concentration $c_{0}$ far from the electrodes is constant. Here, $\mathrm{n}=2$ for divalent electroactive cations, $D$ is their diffusion constant, and $\mathrm{F}=96485 \mathrm{C} \mathrm{mol}^{-1}$ is Faraday's constant. The concentration gradient drives the $\mathrm{M}^{2+}$ ions in the electrolyte towards the cathode, where they are reduced to metal in two single-electron transfer steps. The concentration $c$ of $\mathrm{M}^{2+}$ is thought to be almost zero at the cathode surface. ${ }^{20,26}$

Here we consider the divalent cations $\mathrm{M}^{2+}$ in aqueous solution, where $\mathrm{M}=\mathrm{Co}, \mathrm{Ni}, \mathrm{Cu}$, or $\mathrm{Zn}$. The reduced species $\mathrm{M}^{+}$enjoy a transient existence in the electrical double layer before accepting an electron from the cathode and being reduced to $\mathrm{M}^{0}$ atoms, which coalesce to form the solid metallic electrodeposit. The water of hydration of the solvated divalent ions is shed at the cathode, and a backflow away from the cathode is established. In the case of copper, for example, the hydration number is about $18,{ }^{27}$ so the volume of water liberated is about 40 times the volume of metal deposited.

Our choice of metals is motivated by the desire to distinguish the different species according to their susceptibility in the three different charge states $\mathbf{M}^{2+}, \mathbf{M}^{+}$, and $\mathbf{M}^{0}$, and thereby learn how the magnetic field exerts its influence. Properties of the ions are summarized in Table II, including 
TABLE I. Characteristic dimensionless numbers in an electrochemical cell. ${ }^{\mathrm{a}}$

\begin{tabular}{lcccc}
\hline \hline Reynolds number & $\mathrm{Re}$ & $u l / v$ & 300 & Streamline flow, close to turbulence \\
Peclet number & $\mathrm{Pe}$ & $u l / \mathrm{D}$ & $3 \times 10^{5}$ & Convection dominates diffusion \\
Magnetic Reynolds number & $\mathrm{Rm}$ & $u l / \eta$ & $3 \times 10^{-7}$ & Magnetic field is uninfluenced by flow \\
Hartmann number & $\mathrm{Ha}$ & $B l(\sigma / \rho v)^{1 / 2}$ & 1 & Lorentz force similar to the shear force \\
Interaction parameter & $\mathrm{N}$ & $\sigma B^{2} l / \rho u$ & 0.3 & Lorentz force similar to inertial damping \\
\hline \hline
\end{tabular}

applied magnetic field $B=0.3 \mathrm{~T}$; flow velocity $u=10 \mathrm{~mm} \mathrm{~s}^{-1}$; cell dimension $l=30 \mathrm{~mm}$; diffusion constant $D=10^{-9} \mathrm{~m}^{2} \mathrm{~s}^{-1}$; viscosity $v=10^{-6} \mathrm{~m}^{2} \mathrm{~s}^{-1}$; conductivity $\sigma=1000 \mathrm{Sm}^{-1}$; magnetic diffusivity $\eta=10^{3} \mathrm{~m}^{2} \mathrm{~s}^{-1}$; density $\rho=100 \mathrm{~kg} \mathrm{~m}^{-3}$.

the Curie law paramagnetic susceptibility ${ }^{22} \chi_{\text {mol }}$ of a mole of the $3 d$ ions, and the $\mathrm{Dy}^{3+}$ ions, which we use to create a strongly paramagnetic but nonelectroactive background. The susceptibility $\chi_{1}$ of one-molar solutions are calculated using the diamagnetic susceptibility of water $-9.0 \times 10^{-6}$. These are dimensionless SI susceptibilities, $\chi=M / H$, where $M$ is the induced magnetic moment per unit volume, with units A $\mathrm{m}^{-1}$. Conversions to other definitions of susceptibility and to cgs units can be found in textbooks. ${ }^{28}$

A variety of magnet arrays, which produce nonuniform fields are used to investigate magnetic patterning. Whenever the magnetic field is nonuniform, another force besides the Lorentz force is operative. Known as the Kelvin force or the field gradient force, this may be written in several equivalent forms when the susceptibility is small and isotropic. The nonuniform field $\boldsymbol{H}$ induces a magnetization $\boldsymbol{M}=\chi \boldsymbol{H}$ in the electrolyte, where the susceptibility $\chi$ is expressed as $\chi_{\text {mol }} c+\chi_{\text {water }}$, where $\chi_{\text {mol }}$ is the molar susceptibility and $c$ is the concentration of ions in the electrolyte in $\mathrm{mol} \mathrm{m}^{-3}$. Since $\chi \ll 1$ for the electrolytes used in electrochemistry (Table II), $\boldsymbol{B}$ and $\mu_{0} \boldsymbol{H}$ can be used interchangeably. They are related by the definition $\boldsymbol{H}=\boldsymbol{B} / \mu_{0}-M^{28}$ or

$$
\boldsymbol{B}=\mu_{0}(\boldsymbol{H}+\boldsymbol{M}) .
$$

The difference between the applied field and the internal field is also negligible, and the Kelvin force is given by the expression $\boldsymbol{F}_{\mathrm{K}}=\mu_{0}(\boldsymbol{M} \cdot \boldsymbol{\nabla}) \boldsymbol{H}$. When $\boldsymbol{M}$ is small and parallel to $\boldsymbol{H}$, and the cell current can be neglected as a source of $\boldsymbol{H}$, the vector identity $\nabla(\mathbf{A} \cdot \mathbf{B})=(\mathbf{B} \cdot \boldsymbol{\nabla}) \mathbf{A}+(\mathbf{A} \cdot \boldsymbol{\nabla}) \mathbf{B}+\mathbf{B} \times$ $(\nabla \times \mathbf{A})+\mathbf{A} \times(\nabla \times \mathbf{B})$ can be used to write the field gradient force $\mathrm{F}_{\nabla H}=\mu_{0} \chi H \nabla H$ as

$$
\boldsymbol{F}_{\nabla B}=\left(1 / 2 \mu_{0}\right) \chi_{m} c \nabla B^{2} .
$$

This may exceed the Lorentz force [Eq. (1)] when the electrolyte is paramagnetic. ${ }^{29}$ The ratio of the magnitudes of the two forces is ${ }^{30}$

$$
R=\chi \nabla B / \mu_{o} j
$$

The field gradient force in the vicinity of the electrode in our experiments is comparable in magnitude to the forces driving convection, so it may be expected to influence convection in the diffusion layer. The magnitudes of the forces at play are summarized in Table III. Ultimately, diffusion will prevail, but times for diffusive mixing are quite long in electrochemical cells. For example, for $1 \mathrm{M} \mathrm{Cu}^{2+}$ in water (diffusion constant $D \approx 10^{-9} \mathrm{~m}^{2} \mathrm{~s}^{-1}$ ), the distances given by the one-dimensional diffusion relation

$$
l=(D t)^{1 / 2}
$$

are $100 \mu \mathrm{m}$ after $10 \mathrm{~s}$ and $1 \mathrm{~mm}$ after $1000 \mathrm{~s}$.

Here we give a detailed account of normal and inverse structuring effects of arrays of small cylindrical magnets on the electrodeposits. Experimental variables include the structure of the magnet array, electrolyte composition and concentration, time evolution, field dependence, and the influence of convection. In this way, we are able to build up a step-by-step understanding of the influence of a nonuniform magnetic field on electrodeposition from a paramagnetic electrolyte and the related convective flows.

\section{EXPERIMENTAL METHODS}

Electrodeposits were produced from acidified solutions of the divalent cations $\mathrm{Co}^{2+}\left(3 d^{7}\right), \mathrm{Ni}^{2+}\left(3 d^{8}\right), \mathrm{Cu}^{2+}\left(3 d^{9}\right)$, and $\mathrm{Zn}^{2+}\left(3 d^{10}\right)$. For cobalt and nickel, the divalent ion,

\begin{tabular}{|c|c|c|c|c|}
\hline & Configuration & 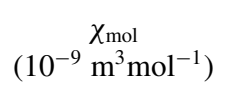 & $\begin{array}{c}\chi_{1}(1-\text { molar }) \\
\left(10^{-6}\right)\end{array}$ & $\begin{array}{c}\mathrm{M}^{2+}+2 \mathrm{e} \rightarrow \mathrm{M}^{(\mathrm{s})} \\
(\mathrm{V} / \mathrm{Ag}-\mathrm{AgCl})^{\mathrm{c}}\end{array}$ \\
\hline $\mathrm{Co}^{2+}$ & $3 d^{7} \mathrm{~S}=3 / 2$ & 78.6 & 69.6 & -0.48 \\
\hline $\mathrm{Ni}^{2+}, \mathrm{Co}^{+}$ & $3 d^{8} \mathrm{~S}=1$ & 41.9 & 32.9 & -0.43 \\
\hline $\mathrm{Cu}^{2+}, \mathrm{Ni}^{+}, \mathrm{Co}^{0 \mathrm{a}}$ & $3 d^{9} \mathrm{~S}=1 / 2$ & 15.7 & 6.7 & +0.14 \\
\hline $\mathrm{Zn}^{2+}, \mathrm{Cu}^{+}, \mathrm{Ni}^{0 \text { a }}$ & $3 d^{10} \mathrm{~S}=0$ & -0.1 & -9.0 & -0.95 \\
\hline $\mathrm{Dy}^{3+}$ & $4 f^{9} \mathbf{J}=15 / 2$ & 594 & 585 & $-2.52^{b}$ \\
\hline
\end{tabular}
monovalent ion and metal atom all have an unfilled $d$ shell and are paramagnetic, whereas for zinc all three are diamagnetic.

TABLE II. Magnetic properties of ions involved in the electrodeposition.

${ }^{\mathrm{a}}$ solid $\mathrm{Co}$ and $\mathrm{Ni}$ are ferromagnetic.

${ }^{\mathrm{b}} \mathrm{M}^{3+}+3 \mathrm{e} \rightarrow \mathrm{M}^{(\mathrm{s})}$.

${ }^{c}$ to convert to the standard hydrogen electrode, add $0.22 \mathrm{~V}$. 
TABLE III. Some forces involved in electrodeposition, units N $\mathrm{m}^{-3}$.

\begin{tabular}{lcc}
\hline \hline Name & Symbol & Force density $\left(\mathrm{N} \mathrm{m}^{-3}\right)$ \\
\hline Lorentz force & $\boldsymbol{j} \times \boldsymbol{B}$ & 10 \\
Field gradient force & $\left(1 / 2 \mu_{0}\right) \chi_{\mathrm{mol}} c \nabla B^{2}$ & 800 \\
Natural convection & $\Delta \rho \mathrm{g}$ & 1000 \\
\hline \hline
\end{tabular}

applied magnetic field $B=0.33 \mathrm{~T}$; field gradient $\nabla B^{2}=200 \mathrm{~T}^{2}$ $\mathrm{m}^{-1}$; current density $j=30 \mathrm{Am}^{-2}$; susceptibility $\chi_{\mathrm{mol}} c=10 \times$ $10^{-6}$; density difference $\Delta \rho=100 \mathrm{~kg} \mathrm{~m}^{-3}$.

In the case of copper, only the divalent ion is paramagnetic (Table I).

\section{A. Electrochemical cell}

The cell used for many of the experiments is shown in Fig. 1(a). The cathode is a foil, either 50- $\mu$ m-thick silver, 50 - $\mu \mathrm{m}$-thick copper, or $17-\mu \mathrm{m}$-thick copper, backed by an array of small cylindrical $\mathrm{Nd}_{2} \mathrm{Fe}_{14} \mathrm{~B}$ permanent magnets. The foil in a particular experiment was chosen to give the better optical contrast. The cell volume is $60 \mathrm{ml}$; it holds $6 \mathrm{mmol}$ of $0.1 \mathrm{M}$ solution, which is sufficient to deposit $\sim 150 \mu \mathrm{m}$ of metal on the cathode. The average thickness of the metal deposits is about $1 \mu \mathrm{m}$, so depletion of the electrolyte in the course of an experiment is insignificant. The cell itself is often oriented with the cathode facing downwards in order to minimize natural convection, but some experiments were done with the cathode vertical, or facing upwards in order to check the effect of convection on the magnetic structuring of the electrodeposits, and to avoid accumulation of hydrogen bubbles on the electrode. A simpler, cylindrical cell with an upward-facing cathode shown in Fig. 1(b) was used in experiments where gravitationally induced convection was negligible. Table IV lists the various electrochemical baths and growth conditions used. The bulk magnetic susceptibilities of the solutions have been calculated from the data in Table II. The diamagnetic corrections for the additives have been neglected.

\section{B. Permanent magnet configurations}

Many different arrangements of the magnets with a diameter of $2 \mathrm{~mm}$, height of $2 \mathrm{~mm}$, and spacing of $3 \mathrm{~mm}$ have

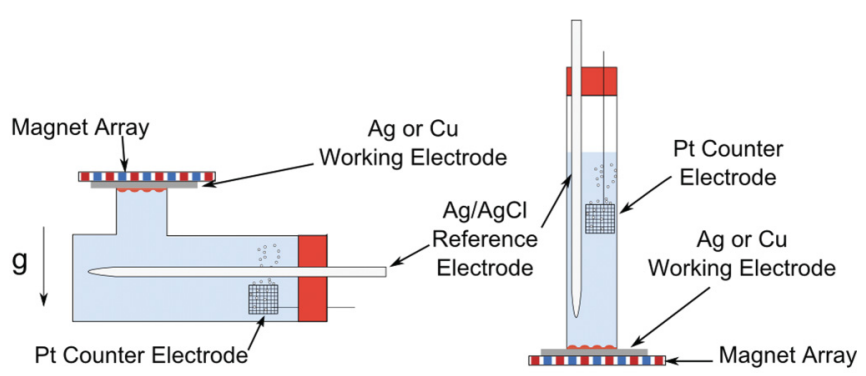

(a)

(b)

FIG. 1. (Color online) Electrochemical cells used for producing magnetically structured deposits. The cathode is a silver or copper foil, backed by an array of permanent magnets, which are outside the cell. (a) The cell on the left is used for different cathode orientations, whereas (b) is used for upward-facing cathodes only. been investigated, including square, triangular, and hexagonal arrays with different patterns of magnetization. We focus mainly on square or hexagonal arrays where the directions of magnetization of adjacent magnets are aligned either parallel or antiparallel. Each array incorporates about 80 magnets, but only 25-30 lie within the circular area of the metal foil of diameter $19 \mathrm{~mm}$ (area $2.8 \mathrm{~cm}^{2}$ ), which was exposed to the electrolyte, in order to minimize magnetic edge effects. The distance between the upper faces of the magnets and the active cathode surface is approximately $250 \mu \mathrm{m}$.

Figure 2 shows contour plots of the distribution of magnetic field $250 \mu \mathrm{m}$ above the surface of some of the magnet arrays. The parallel arrays lead to circumferential hydrodynamic flow around the perimeter of the cathode driven by the Lorentz force [Eq. (1)], whereas the antiparallel arrays produce unfrustrated alternating circular flows around each magnet. ${ }^{24}$

\section{Deposit characterization}

All the deposits were photographed using a (DNT GmbH) 1.3-megapixel charge-coupled device (CCD) digital microscope USB camera. Some thickness profiles were determined using a Dektak 6M. Many were examined by scanning electron microscopy (SEM), and in some cases the profiles were observed directly in cross sections produced by milling with a focused $\mathrm{Ga}$ ion beam (FIB), using an FEI Strata 256 instrument.

\section{RESULTS}

We present the results in two sections; the first is devoted to direct patterning, where the electrolyte is a solution of the electroactive $3 d$ ion, and the second is devoted to inverse patterning from paramagnetic or diamagnetic solutions of electroactive $3 d$ ions in an electrolyte containing a high background concentration of strongly paramagnetic but nonelectroactive $\mathrm{Dy}^{3+}$ ions. In view of the complexity of data, we present results in a series of subsections where one variable is altered at a time, and inferences are drawn from the data at each step.

\section{A. Direct patterning \\ 1. Different metals}

Figure 3 shows patterns obtained for $1 \mathrm{M}$ solutions of $\mathrm{Co}$, $\mathrm{Ni}, \mathrm{Cu}$, and $\mathrm{Zn}$ with a downward facing cathode backed by an alternating hexagonal magnet array. The deposition time is 600 or $1200 \mathrm{~s}$. The deposits of the first three metals are clearly structured in a way that reflects the fields produced by the alternating hexagonal array. The patterning effect is also present when the magnets are all parallel, and the patterns are often clearer when the deposition is galvanostatically controlled, especially for the ferromagnetic metals, Co and Ni. Little sign of structuring was observed for zinc. The zinc deposits were practically uniform in thickness, but closer inspection revealed some difference in texture in the vicinity of the magnets, suggesting an influence of the magnetic field on the nucleation process. Further evidence for a magnetic influence on nucleation and early-stage crystal growth is an increase of the short-time transient current after applying the potential to initiate deposition. A first conclusion from the data in Fig. 3 is that it is the paramagnetic character of the divalent 
TABLE IV. Solutions and growth parameters used.

\begin{tabular}{|c|c|c|c|c|}
\hline Bath & Content & Control $^{\mathrm{a}}$ & $\begin{array}{c}E \text { or } I \\
(\mathrm{~V}) \text { or }(\mathrm{mA})\end{array}$ & Time (min) \\
\hline (A) & $1 \mathrm{M} \mathrm{ZnCl}_{2}+0.4 \mathrm{M} \mathrm{H}_{3} \mathrm{BO}_{3}$ & $\mathrm{P}$ & $-2.0 \mathrm{~V}$ & 10 \\
\hline (B) & $2 \mathrm{M} \mathrm{CoSO}_{4}+0.6 \mathrm{M} \mathrm{H}_{3} \mathrm{BO}_{3}$ & $\mathrm{P}$ & $-1.2 \mathrm{~V}$ & 20 \\
\hline$\left(\mathrm{B}^{\prime}\right)$ & $1 \mathrm{M} \mathrm{CoSO}_{4}+0.4 \mathrm{M} \mathrm{H}_{3} \mathrm{BO}_{3}$ & G & $-5 \mathrm{~mA}$ & 10 \\
\hline (C) & $1 \mathrm{M} \mathrm{NiSO}_{4}+0.4 \mathrm{M} \mathrm{H}_{3} \mathrm{BO}_{3}$ & G & $-5 \mathrm{~mA}$ & 10 \\
\hline (D) & $\begin{array}{c}1 \mathrm{M} \mathrm{CuSO}_{4}+1.2 \mathrm{M} \mathrm{H}_{2} \mathrm{SO}_{4} \\
+0.25 \mathrm{mM} \mathrm{KCl}\end{array}$ & $\mathrm{P}$ & $-0.4 \mathrm{~V}$ & 20 \\
\hline$(\mathrm{E})$ & $\begin{array}{c}0.1 \mathrm{M} \mathrm{CuSO}_{4}+1 \mathrm{M} \mathrm{NaSO}_{4} \\
+0.1 \mathrm{M} \mathrm{CH}_{3} \mathrm{COOH}+0.1 \mathrm{M} \mathrm{CH}_{3} \mathrm{COONa}\end{array}$ & $\mathrm{P}$ & $-0.85 \mathrm{~V}$ & 10 \\
\hline$(\mathrm{F})$ & $0.1 \mathrm{M} \mathrm{CuSO}_{4}+1 \mathrm{M} \mathrm{DyCl}_{3}+0.4 \mathrm{M} \mathrm{H}_{3} \mathrm{BO}_{3}$ & G & $-5 \mathrm{~mA}$ & 20 \\
\hline (G) & $\begin{array}{c}0.545 \mathrm{M} \mathrm{CuSO}_{4}+0.25 \mathrm{mM} \mathrm{NaCl} \\
+1.2 \mathrm{M} \mathrm{H}_{2} \mathrm{SO}_{4}\end{array}$ & G & $-10 \mathrm{~mA}$ & 10 \\
\hline$(\mathrm{H})$ & $\begin{array}{c}0.1 \mathrm{M} \mathrm{ZnCl}_{2}+1 \mathrm{M} \mathrm{DyCl}_{3} \\
+0.4 \mathrm{M} \mathrm{H}_{3} \mathrm{BO}_{3}\end{array}$ & $\mathrm{P}$ & $-1.2 \mathrm{~V}$ & 10 \\
\hline
\end{tabular}

${ }^{\mathrm{a}} \mathrm{P}$ denotes potentiostatic control, and $\mathrm{G}$ denotes galvanostatic control.

cation in solution that is largely responsible for the patterning in a magnetic field.

It is not immediately obvious how the optical contrast and colors in images like those of Fig. 3 are to be interpreted. We, therefore, examined the deposits in the scanning electron microscope and excised 20- $\times 5$ - $\mu$ m-rectangular trenches in the samples, which were about $5 \mu \mathrm{m}$ deep, using an FIB so that the cross sections could be examined, and the average deposit thickness on the copper or silver substrate determined. Many of the deposits were too rough to characterize by atomic force microscopy, and Dektak scans were often very noisy. ${ }^{24}$

For the cobalt deposits, it can be seen in Fig. 4(a) that the deposits beneath the magnets, which appear bright in the optical image are smoother and thinner than those between the magnets, which appear duller and show an angular texture of crystallites on a submicron scale, which acts as an effective scatterer of light.

In the case of copper, illustrated in Fig. 4(b), the regions between the magnets are smoother, and the regions beneath

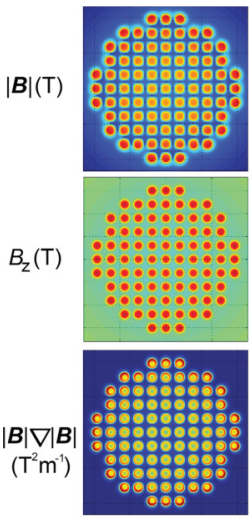

(a)

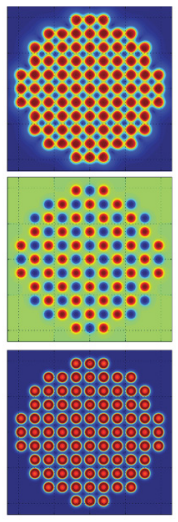

(b)

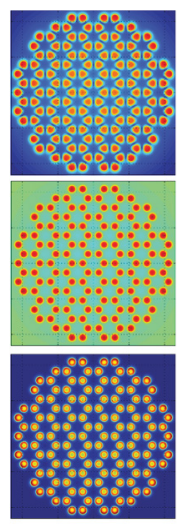

(c)

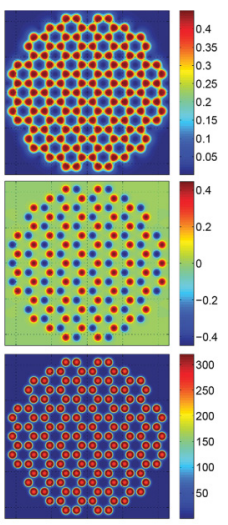

(d)
FIG. 2. (Color online) Magnet arrays used for the magnetoelectrodeposition, showing contours of $|\boldsymbol{B}|, B_{\mathrm{z}}$, and $|\boldsymbol{B}| \nabla|\boldsymbol{B}|$ in the three rows. The columns are (a) square parallel, (b) square alternating, (c) hexagonal parallel, and (d) hexagonal alternating arrangements. $|\boldsymbol{B}|$ and $B_{\mathrm{z}}$ are plotted in $\mathrm{T} ;|\boldsymbol{B}| \nabla|\boldsymbol{B}|$ is in $\mathrm{T}^{2} \mathrm{~m}^{-1}$. the magnets are thicker and exhibit a rough, corncob structure, which scatters the light. The average thickness between the magnets is $0.45 \pm 0.05 \mu \mathrm{m}$, whereas that in the $400-\mathrm{mT}$ field directly beneath the magnets is $1.8 \pm 0.1 \mu \mathrm{m}$, although the deposit is not fully dense.

In the next sections we focus on copper, so as to avoid any effects of deformation of the applied magnetic field by a ferromagnetic electrodeposit.

\section{Concentration and susceptibility}

Next we investigate how the susceptibility of the electrolyte influences the structuring. Water is diamagnetic, with $\chi=$ $-9.0 \times 10^{-6}$. The Curie law contribution for a mole of the dissolved copper ions is $15.7 \times 10^{-6}$. Hence, a $570 \mathrm{mM}$ copper solution will have no net susceptibility. The additives will change the zero-susceptibility concentration by a negligible amount. Figure 5 compares deposits for copper electrolytes with positive and negative net susceptibility. There is little difference in their overall structure, which reflects the magnet array, regardless of the overall sign of the susceptibility. The deposit from the $10 \mathrm{mM}$ solution shows an unstructured patch of copper at the center, which may reflect an established pattern of convection where the return flow is downwards towards the center. The small, submillimeter dots are due to nucleation at defects in the surface of the silver substrate. The conclusion from these data is that it is the susceptibility of the electroactive species that governs the deposition, not the overall susceptibility of the solution. This finding is in accord with other investigations. ${ }^{23}$

\section{Time dependence}

The time dependence of the patterned electrodeposition was studied in some detail for the $100 \mathrm{mM}$ copper solution, as illustrated in Fig. 6. There the current transient is plotted with and without the magnet array, and a series of images of the deposits is shown after various times. No patterning is observed in the first 10-20 s, but patterns appear thereafter. In the initial stages, the deposition is rapid, because the concentration of $\mathrm{Cu}^{2+}$ close to the cathode is large; the diffusion layer is 

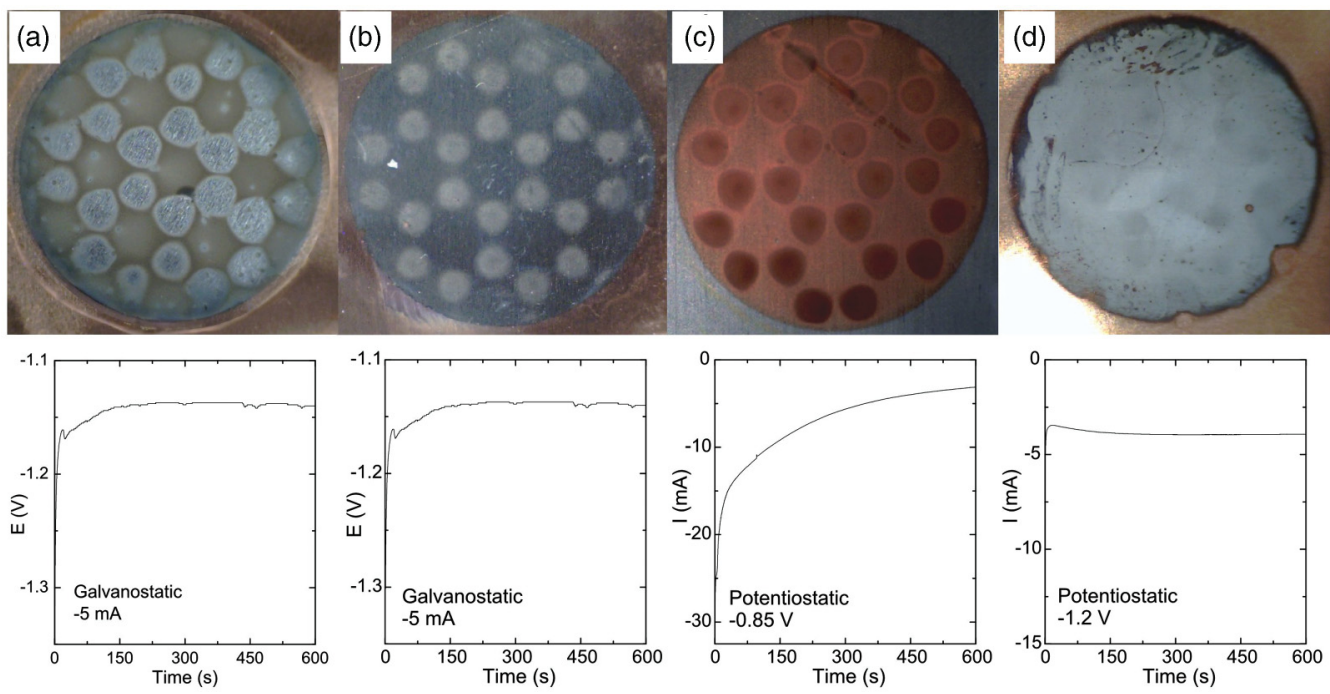

FIG. 3. (Color online) Deposits with hexagonal alternating magnet arrays for (a) Co (bath $\mathrm{B}$ at $-5 \mathrm{~mA}$ for $600 \mathrm{~s}$ ), (b) $\mathrm{Ni}$ (bath $\mathrm{C}$ at $-5 \mathrm{~mA}$ for $600 \mathrm{~s}$ ), (c) $\mathrm{Cu}$ (bath $\mathrm{E}$ at $-0.85 \mathrm{~V}$ for $600 \mathrm{~s}$ ), and (d) $\mathrm{Zn}$ (bath $\mathrm{A}$ at $-1.2 \mathrm{~V}$ for $600 \mathrm{~s}$ ).

evolving quickly with time. The long-time evolution of current varies as $t^{-1 / 2}$, as shown in the insert. Fitting the slope here to the Cottrell equation ${ }^{31}$

$$
j(t)=\mathrm{nF} D^{1 / 2} c_{0}(\pi t)^{-1 / 2}
$$

gives $D=0.510^{-9} \mathrm{~m}^{2} \mathrm{~s}^{-1}$. With the downward-facing cathode, the steady state is only achieved after several hundred seconds.

In this case, the charge passed in the first $15 \mathrm{~s}$, before traces of the pattern appear is $\sim 0.4 \mathrm{C}$, which corresponds to a layer thickness of $\sim 50 \mathrm{~nm}$. After $1000 \mathrm{~s}$, the current density is almost constant in time, and the $5.2 \mathrm{C}$ charge passed, corresponds to an average deposit thickness of $0.6 \mu \mathrm{m}$. The copper dots only cover about $20 \%$ of the surface areas, so the charge passed is quite consistent with the structure of the copper electrodeposit seen in Fig. 4(b), where a trace of the dense uniform layer is seen to underlie the corncob deposits beneath the magnets.
The conclusion here is that the patterns develop only after the time needed to set up the diffusion layer.

\section{Different arrays}

Next, we examine the deposits obtained after $600 \mathrm{~s}$ for different magnet arrays. The idea is to compare them with the profiles of $|\boldsymbol{B}|, B_{z}$, and $\nabla B^{2}$ in Fig. 2, in order to see if we can decide which one determines the form of the deposit. A difference between the alternating and aligned arrays is the presence or absence of a perpendicular field in the interstitial space between the magnets. This is best seen in the $B_{\mathrm{z}}$ profiles for the hexagonal arrays in Fig. 2. On examining the deposited profiles in Fig. 7, we see that the shape of the dots in the structured deposits appears to follow the contours of $|\boldsymbol{B}|$. This is further strengthened by the lower panel in Fig. 7, showing the predicted diffusion layer modulation from the magnetic pressure model discussed later using Eq. (12).

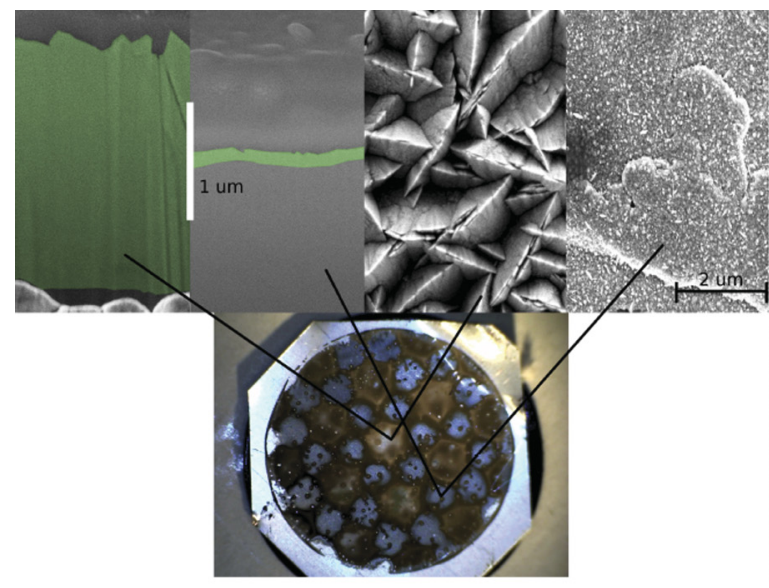

(a)

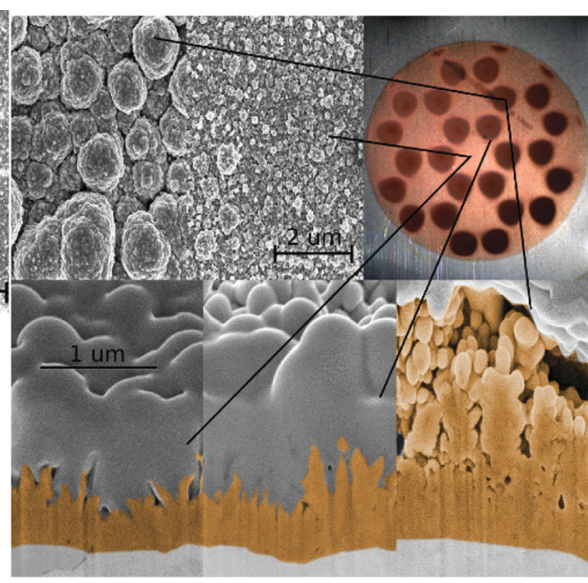

(b)

FIG. 4. (Color online) SEM images of the surfaces and cross sections of (a) Co and (b) Cu electrodeposits. In each pair, the image on the left shows an area between the magnets, where the field is $<10 \mathrm{mT}$, while the image on the right shows an area directly below a magnet, where the field is about $400 \mathrm{mT}$. 


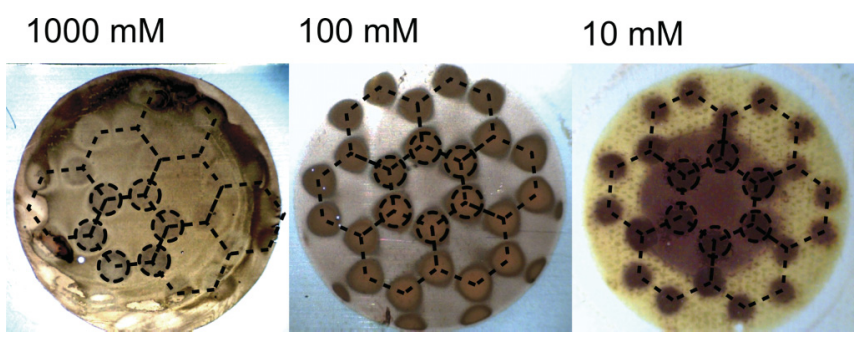

FIG. 5. (Color online) Electrodeposits after $600 \mathrm{~s}$ from copper solutions with three different molarities. The overall susceptibility of the electrolyte is positive for the first panel and negative for the other two. Black hexagons and circles highlight the periodicity of the deposits.

The time dependence of the current is distinctly different for the various arrays. Currents are greater in the initial stages for the alternating arrays, as shown in Fig. 8. This is confirmed by the potential variation in galvanostatic scans. When the diffusion layer is set up at longer times, the current becomes independent of the array. The effect may be due to the early action of the field gradient force during the transient, or to the action of the Lorentz force, which depends on the horizontal component of the field. In the early stages of growth, the magnetic field experienced by the diffusion layer is much greater than when it reaches its fullest extent at steady state. Similarly, the magnetic fields below the alternating arrays are, on average, more intense than the magnet fields below the parallel arrays (see Fig. 2).

The conclusion is that the alternating arrays generate larger currents in the transient regime, although when the diffusion layer is fully established the current becomes independent of the array.

\section{Effects of convection}

The influence of natural convection on the deposits is nicely illustrated by comparing deposits obtained with the cell oriented with the cathode facing down, facing up, or vertical. The structure is largely destroyed by natural convection when the cathode is facing upwards, and there is streaking of the deposits when the cathode is vertical (Fig. 9). The lower panels show how the limiting current density is enhanced by convection, and the red curves obtained with no copper in the bath indicate that the hydrogen current here is negligible. The force density involved in natural convection is of the order $\Delta \rho \mathrm{g}$, where $\Delta \rho$ is the density difference between electrolytes when it is concentrated or depleted in the electroactive species. The density difference for a $0.1 \mathrm{M}$ copper solution is about $15 \mathrm{~kg} \mathrm{~m}^{-3}$, and the force density is about $150 \mathrm{~N} \mathrm{~m}^{-3}$.

The conclusion here is that the force densities involved in the diffusion layer, which lead to patterning, are comparable to those in natural convection, namely of order, $10^{2} \mathrm{Nm}^{-2}$.

\section{Influence of a uniform applied field}

It is possible to place the cell in a uniform field produced by an external magnet. We have examined the effect of the external field when it is applied parallel or perpendicular to the axis of the magnet array. The anisotropy field of the Nd-Fe-B magnets is $7.7 \mathrm{~T}$, and their coercivity is $1.2 \mathrm{~T}$, so an external field of $0.5 \mathrm{~T}$ has little influence on the field produced by the magnet array. The external field will induce an additional magnetization in the paramagnetic solution, and it modifies the field pattern at the cathode surface. The $0.1 \mathrm{M} \mathrm{Cu}$ solution was again used, as this provides some of the clearest deposits.

When the parallel field adds to that produced by the magnets in an aligned array [Fig. 10(c)], the effect is to augment the

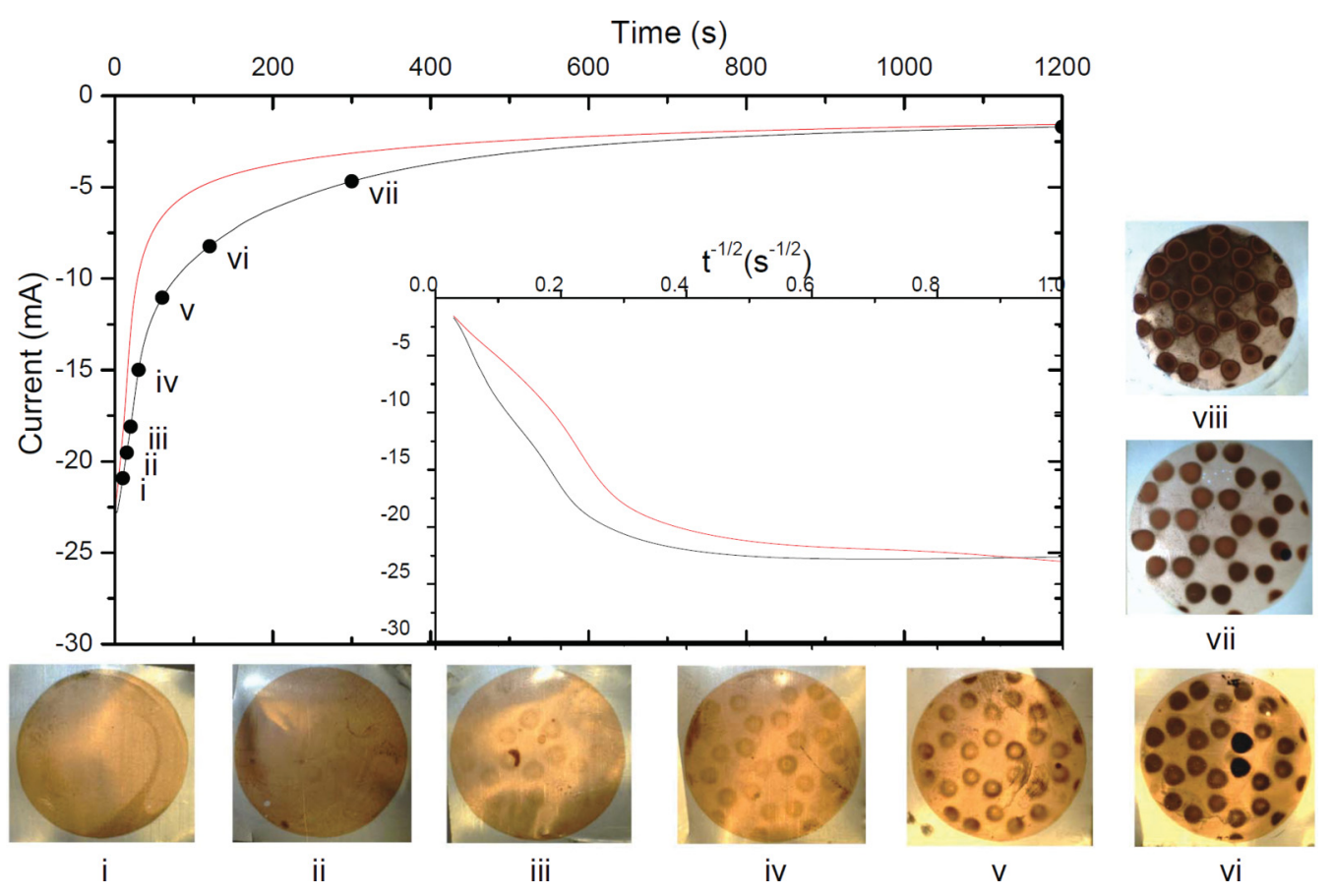

FIG. 6. (Color online) Time dependence of the current from a 100-mM copper solution with a downward-facing electrode, with and without the hexagonal alternating magnet array. The images show the patterns after different deposition times. The current trace without the magnets is shown by the red (medium gray) line. The Cotrell plot is shown as an insert. 

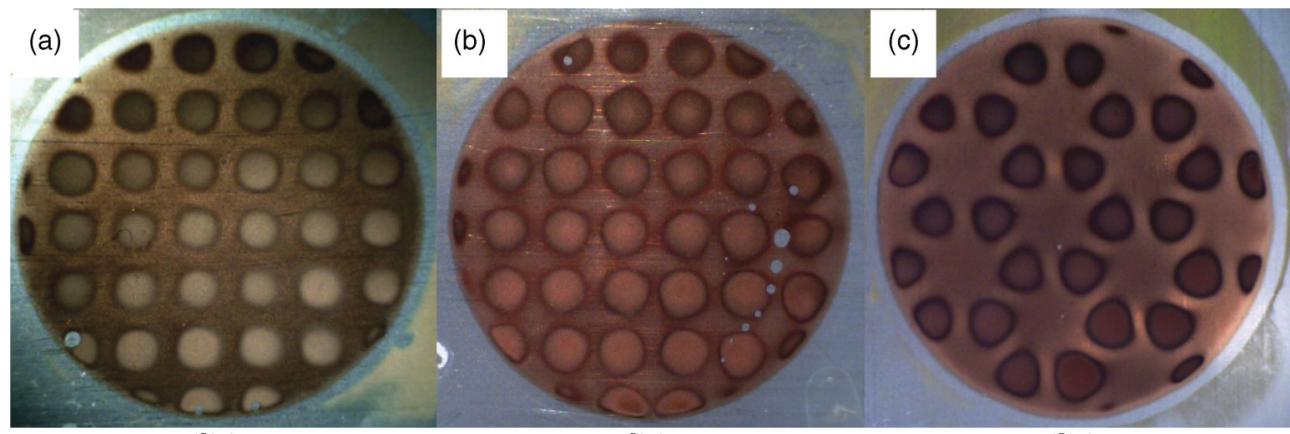

(d)
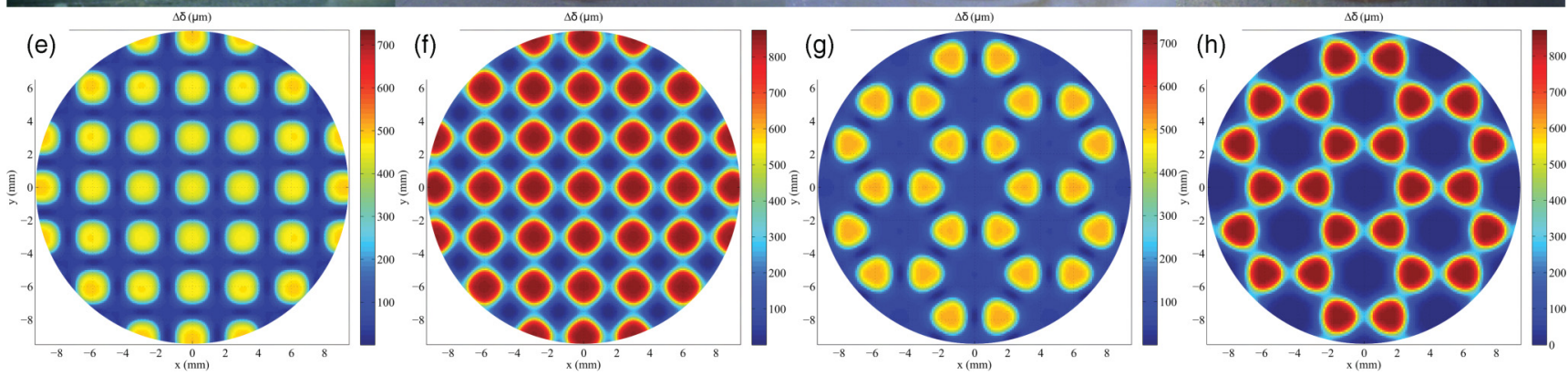

FIG. 7. (Color online) Top panel: structured electrodeposits obtained from $0.1 \mathrm{M}$ copper solution with (a) square parallel, (b) square alternating, (c) hexagonal parallel, and (d) hexagonal alternating arrays. Bottom panel: corresponding diffusion layer thickness modulation.

contrast of the deposit beneath the magnets. When the cathode is facing upwards, the effects of convection modified by the Lorentz or field gradient force come into play. Some beautiful chiral patterns of the copper electrodeposits are then observed; Figs. 10(d) and 10(f) show the patterns for an upwardfacing parallel array with a parallel or antiparallel 500-mT field.

When the field opposes that produced by the aligned array, it increases the field in the interstitial space and reduces that beneath the magnets. This has the effect of increasing deposition between the magnets, leading to inverse patterning [Fig. 11(a)]. When the alternating array is used, the deposit is thicker in the regions where the applied field at the cathode reinforces that of the permanent magnets-the dots become much bigger - and it is thin when the field subtracts from that of the magnets. The number of dots is halved [Fig. 10(g)]. The effect of a horizontal field is to extend the deposits in-plane, in the perpendicular direction.

We conclude that it is possible to reinforce or reverse the patterning effect of the magnet array by adding a uniform vertical field. The pattern is controlled by the component of field normal to the electrode surface.

\section{B. Inverse patterning}

The results we have presented so far indicate that the patterning effect stems from the inhomogeneous magnetic field acting on paramagnetic electroactive ions in the electrolyte, especially the diffusion layer. There is no net force to first order when a uniform magnetic field acts on an electrolyte whose susceptibility is nonuniform, provided $\chi \ll 1 .{ }^{32}$

Now we explore the effects when a strongly paramagnetic but nonelectroactive species is added to the electrolyte. Recent reports have shown that inverse patterning can be achieved in these circumstances. ${ }^{24,25}$ In most of our experiments, $1 \mathrm{M}$ dysprosium, which has $\chi_{1}=594 \times 10^{-6}$, is added to the electrolyte solution. Strong magnetic contrast thereby results between the electroactive species $\left(\mathrm{Cu}^{2+}\right.$ or $\left.\mathrm{Zn}^{2+}\right)$ and the nonelectroactive background. Any weakly paramagnetic or diamagnetic material immersed in a concentrated dysprosium solution behaves as if it has a very strong diamagnetic susceptibility. Almost any solid so immersed can be levitated in the magnetic field of a laboratory electromagnet. ${ }^{33}$ However, we also observe striking magnetic field effects with much weaker Dy solutions, where the susceptibility of the copper is similar to that of the dysprosium.

It should also be noted that two dysprosium salts were considered, $\mathrm{DyCl}_{3}$, and $\mathrm{Dy}\left(\mathrm{NO}_{3}\right)_{3}$. The nitrate ions from $\mathrm{Dy}\left(\mathrm{NO}_{3}\right)_{3}$ solutions were found to block the electrode surface due to the formation of insulating oxide layers such as zinc oxide. ${ }^{34}$ This was confirmed by replacing $\mathrm{Dy}\left(\mathrm{NO}_{3}\right)_{3}$ with $\mathrm{KNO}_{3}$, and no such blocking issues were found for $\mathrm{DyCl}_{3}$.

\section{Different materials}

Patterning of copper and zinc is illustrated in Fig. 11 for a downward-facing, alternating hexagonal array. In each case, a $0.1 \mathrm{M}$ solution of $\mathrm{M}^{2+}$ is mixed with $1 \mathrm{M} \mathrm{Dy}^{3+}$. The deposit builds up mainly in the interstitial space between the magnets where the field is low. The thin copper deposits beneath the magnets consist of isolated crystallites about a micron in size, which do not completely cover the substrate, whereas the deposits in the interstitial space are thicker with a distinct dendritic or fernlike morphology. Increasing the current density or overpotential makes the copper deposit in the interstitial space increasingly rough and 

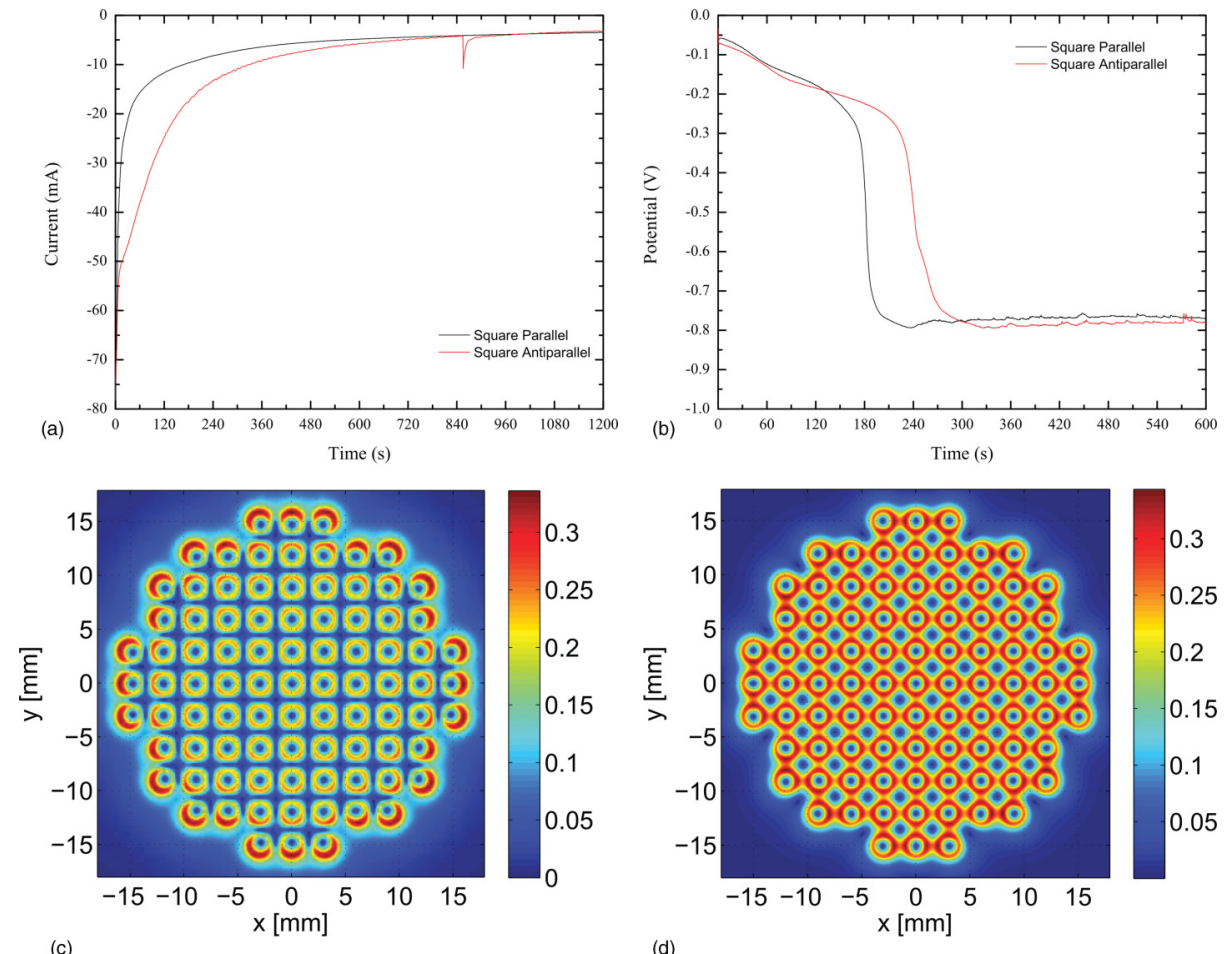

FIG. 8. (Color online) Time dependence of (a) the copper current at $-0.85 \mathrm{~V}$ and (b) the potential at -20 $\mathrm{mA}$ at the downward-facing electrode for parallel and alternating square magnet arrays compare to the response with a single magnet. Panels (c) and (d) compare the magnitude of the horizontal component of the magnetic field for the parallel and alternating arrays. powdery, while the regions beneath the magnets remain shiny and smooth. Less distinct inverse patterning was found for Co or Ni.

The inverse deposits for the corresponding Zn/Dy bath are exceptionally well defined in the optical images. The shiny areas between the magnets are covered in a carpet of 100- to 200-nm-hexagonal zinc platelets, which is about $100 \mathrm{~nm}$ thick, whereas the interstitial areas are much rougher, composed of thicker stacks of edge-on hexagonal $\mathrm{Zn}$ crystallites, which are about $1 \mu \mathrm{m}$ in size and 50 to $100 \mathrm{~nm}$ thick.

Zinc is a diamagnetic metal, with a susceptibility of $-16 \times$ $10^{-6}$, which grows in the form of platelets perpendicular to the hexagonal $c$ axis. The demagnetizing factor of these platelets $N \sim 1$ gives them a slight preference to orient perpendicular to an applied field. The shape anisotropy energy of $\mathrm{Zn}$ in $300 \mathrm{mT}$ is only of order $1 \mathrm{~J} \mathrm{~m}^{-3}$. The energy is very small, but it seems that it is sufficient to influence the metal nucleation and growth.

We conclude that the main factor governing the structure of the deposits is now the susceptibility of the nonelectroactive dysprosium, not the electroactive species as in Sec. III A.

\section{Different arrays}

Some examples of the inverse zinc patterns obtained after $300 \mathrm{~s}$ are illustrated in Fig. 12. The structures are notably different for the parallel and alternating arrays, which may be reinforced by the tendency of the zinc crystallites to grow perpendicular to the local magnetic field. A comparison with the top row in Fig. 2 suggests that it is the magnitude of the magnetic field that determines the pattern of the zinc deposits.

\section{Time dependence}

The time dependence of the current density for $0.1 \mathrm{M}$ zinc + $1 \mathrm{M}$ dysprosium with and without the magnet arrays are shown in Fig. 13, together with a series of pictures, which illustrate how the pattern develops with time. There may be a period of about 1 to $2 \mathrm{~s}$ at the beginning when a large current flows, yet no distinct patterning of the zinc electrodeposit is evident, but patterns are evident from $4 \mathrm{~s}$. There is a period around $7-15 \mathrm{~s}$ when a vortexlike pattern appears as the growth mode changes to one where a Mercedes logo appears at the center of each bright hexagon. There is no such feature in any of the field profiles, which indicates that it is convective in origin. It disappears after $300 \mathrm{~s}$. The mixed region corresponds to the times when the current transient is not entirely reproducible, but falls in an envelope defined by the two extreme traces. In some of the deposits, a pattern of whorls suggests a stable pattern of convection at the surface. A time of $15 \mathrm{~s}$ corresponds to a charge passed of $0.4 \mathrm{C}$, which translates to depletion of the $\mathrm{Zn}$ to a depth of $70 \mu \mathrm{m}$, deposition of a layer of $\mathrm{Zn} 65 \mathrm{~nm}$ thick, and shedding of a layer of water almost three microns deep, assuming 18 water molecules are associated with the zinc hydration sphere. ${ }^{27}$

The slope of the Cottrell plot in Fig. 13 gives a diffusion constant $D=3.0 \times 10^{-9} \mathrm{~m}^{2} \mathrm{~s}^{-1}$; this is close to the value expected for $\mathrm{Zn}$. The diffusion constant of Dy is lower, approximately $0.6 \times 10^{-9} \mathrm{~m}^{2} \mathrm{~s}^{-1} \cdot 35$

The changes in growth mode of the inverse deposits are illustrated by the galvanostatic data on copper taken at $-5 \mathrm{~mA}$ in Fig. 14. Unlike the direct deposits, where a single step is seen in the galvanostatic potential is needed to maintain the current [Fig. 8(b)], here there may be two or three steps. The first, from -0.1 to $-0.3 \mathrm{~V}$ occurs when about $1 \mathrm{C}$ has passed. During this early time period, the growth is sporadic, and almost no 


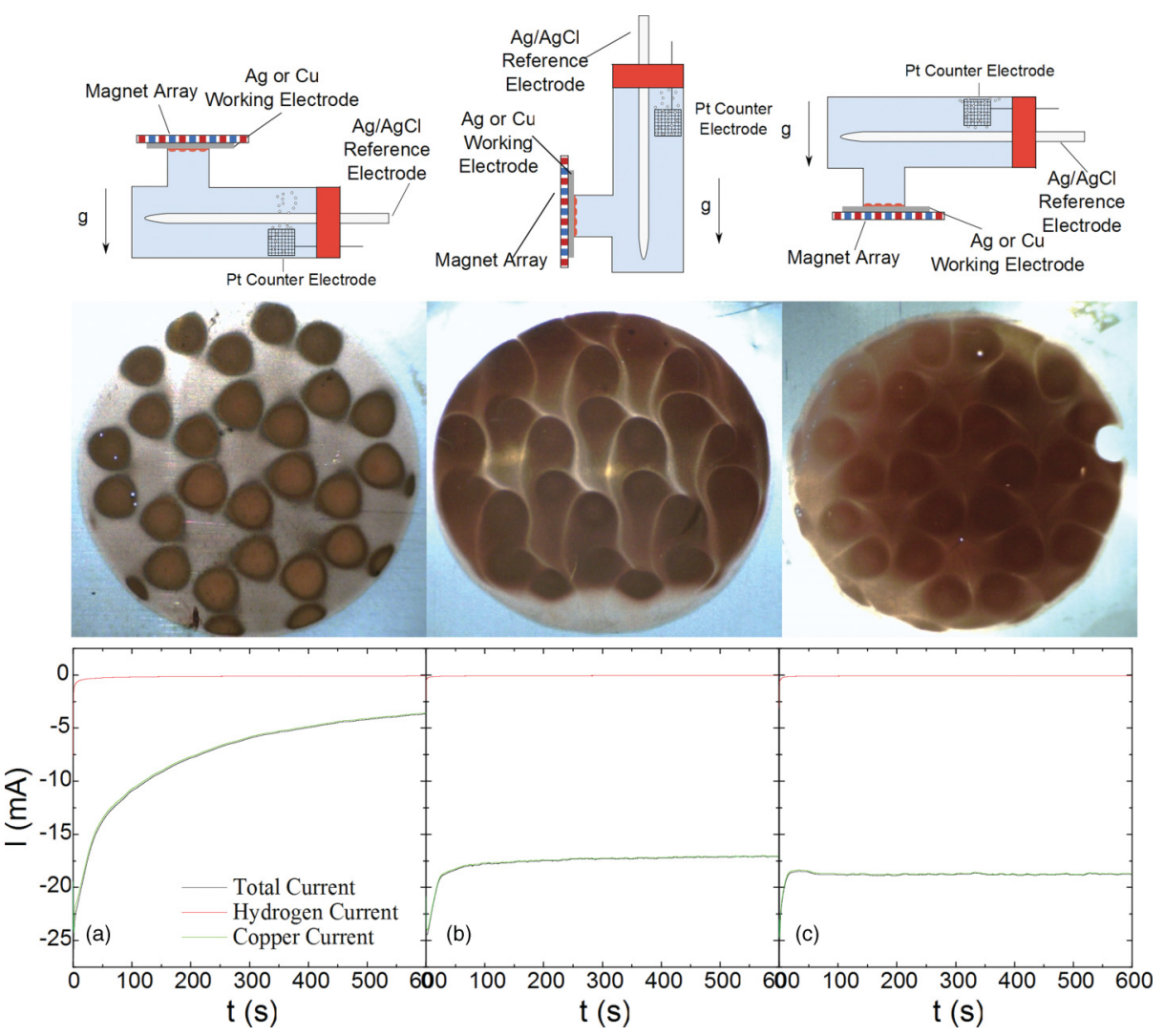

FIG. 9. (Color online) Patterns obtained with a $0.1 \mathrm{M}$ copper solution (bath E) with the cathode facing (a) downwards, (b) vertically, or (c) upwards. The lower panels show the time dependence of the current in the three configurations. The red (medium gray) curves show the hydrogen currents, obtained with no copper in the bath.

$\mathrm{Cu}$ seems to be deposited on the surface. However, during the potential step to $-0.3 \mathrm{~V}$, a smooth $\mathrm{Cu}$ layer rapidly deposits on the surface, which is related to the main current transient in the potentiostatic scans. A second step to $-0.8 \mathrm{~V}$ occurs after about $1.5 \mathrm{C}$ without magnets, and at 3 to $4 \mathrm{C}$ when the magnet array is present. The powdery, dendritic growth of copper takes place at this high overpotential. There is then a return to an overpotential of $-0.3 \mathrm{~V}$ and a smoother growth mode after $2.3 \mathrm{C}$ without magnets and after 4-5 $\mathrm{C}$, when a parallel array is used. This was not observed for the alternating arrays, where the overpotential remains high, and the growth is dendritic.

The main conclusion is that the patterning here is again dependent on the depletion of the electroactive species in a diffusion layer, but that local convection plays a role in stabilizing different growth modes, which were not encountered for the normal deposits.

\section{Effects of convection}

The influence of natural convection on the inversely structured deposits is again illustrated by comparing the zinc deposits obtained at $-1.2 \mathrm{~V}$ with the cell positioned with the cathode facing down, facing up, or vertical. The structure persists when the cathode is facing upwards (Fig. 15), or when it is vertical, and there is no sign of a vertical elongation of the deposits. Little zinc is deposited under the magnets whatever the orientation, but judging from the charge passed, the zinc deposit between the magnets is twice as thick for the vertical and upward-facing arrays, where the mass transport is aided by natural convection. At the potential used, there is also a significant hydrogen current, but the hydrogen evolution does not disrupt the patterning. The inverse structures, therefore, appear to be considerably more stable in the presence of natural convection than the direct, copper structures, shown in Fig. 9. The initial transient before quasiequilibrium conditions are established lasts only $5 \mathrm{~s}$ for the downward-facing cathode, but $50 \mathrm{~s}$ in the upward or vertical orientations.

The integrated current curve (Fig. 16) shows the charge deposited in the transient and steady states. Approximately $1 \mathrm{C}$ is associated with the transient, which corresponds to a zinc deposit of thickness $\sim 100 \mathrm{~nm}$, taking into account the current efficiency and the limited area where the zinc is deposited.

The conclusion here is that the force densities, which lead to inverse patterning, are stronger than those involved in natural convection, that is $\gg 10^{2} \mathrm{~N} \mathrm{~m}^{-3}$.

\section{Effect of a uniform field}

The effect of a uniform external field on the deposits from the $\mathrm{Zn} / \mathrm{Dy}$ solution was examined by placing the cell of Fig. 1(b) in the vertical room-temperature bore of a superconducting magnet or a $0.5-\mathrm{T}$ Halbach cylinder and examining the deposits from parallel hexagonal arrays, when the applied field is parallel or antiparallel to the magnetization direction. In the first case, the fields above and between the magnets are approximately 800 and $300 \mathrm{mT}$, respectively, but in the second case, they are -200 and $-700 \mathrm{mT}$. It is clear from Fig. 17 that the deposits in the $+500-\mathrm{mT}$ field are similar to those in zero field, while those in $-500 \mathrm{mT}$ are reversed, i.e., the zinc is deposited above the magnets, as for the normal deposits in Sec. III A. 

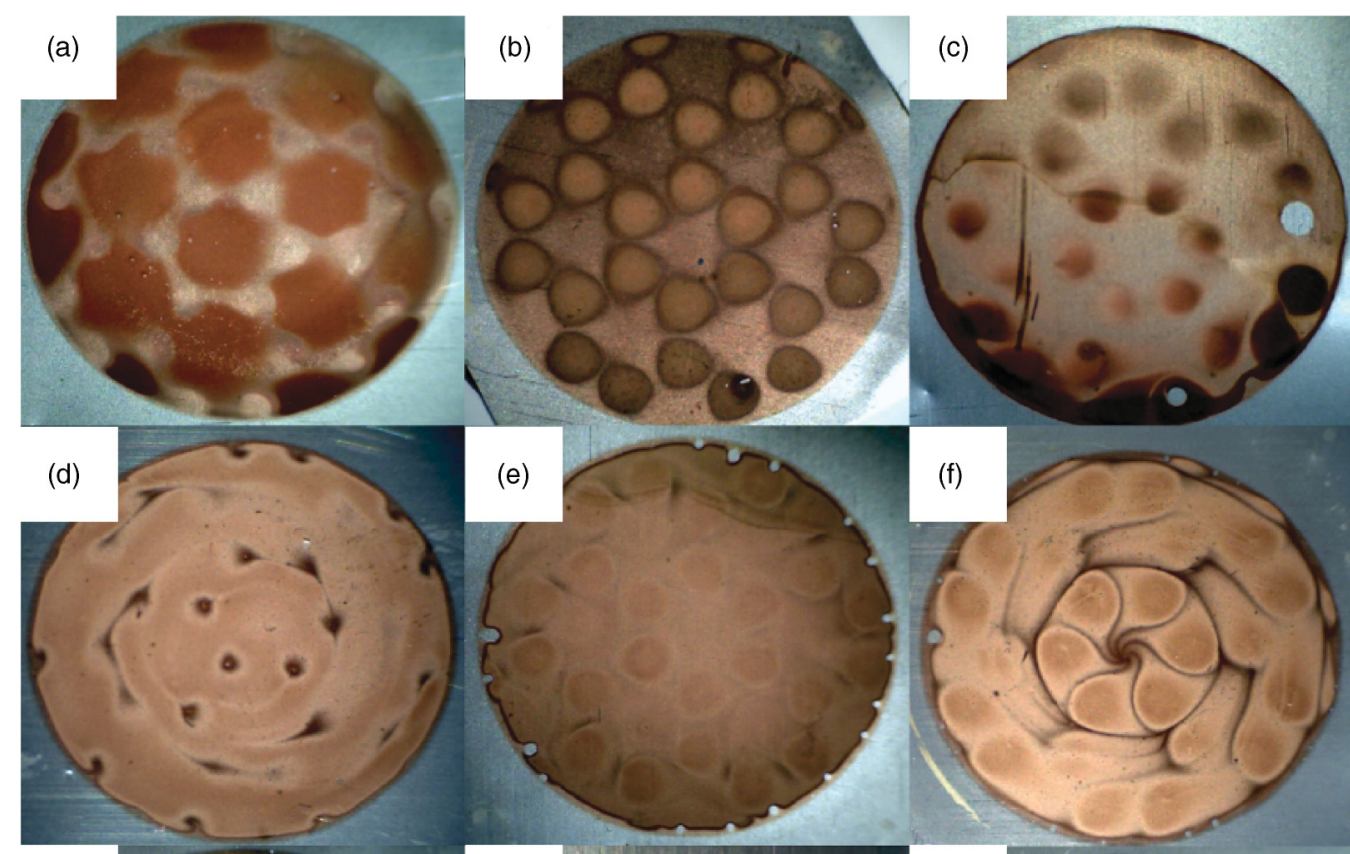

(g)

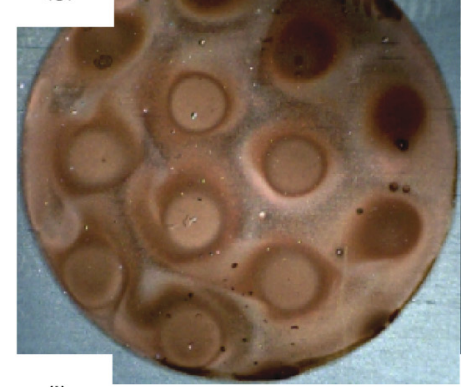

(j)

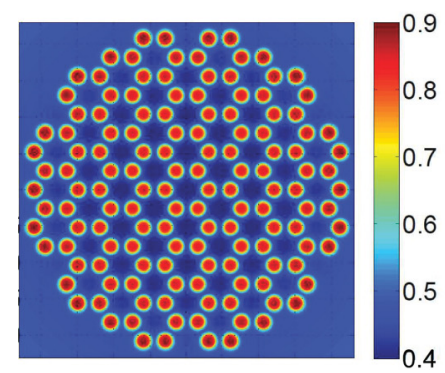

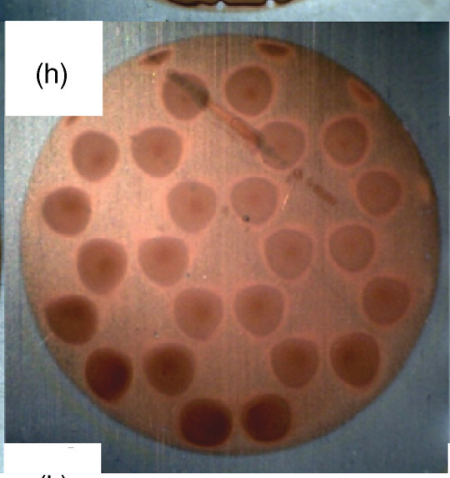

(k)

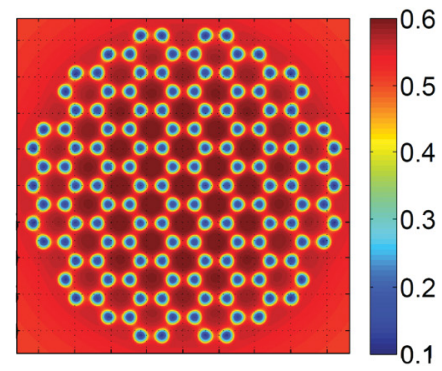

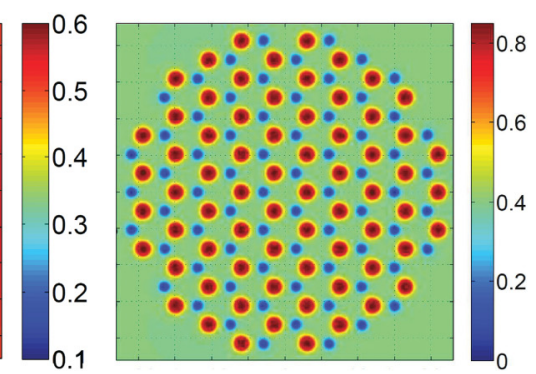

(I)

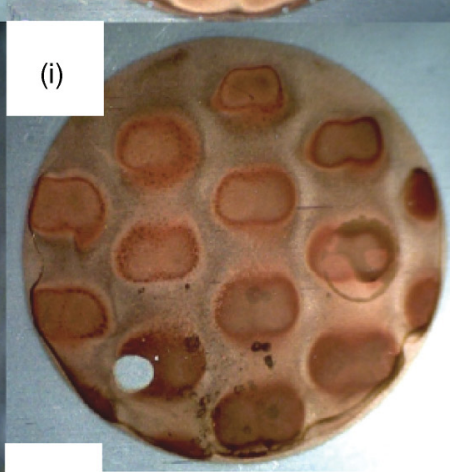

(I)

FIG. 10. (Color online) Patterns produced from the $0.1 \mathrm{M}$ copper bath by hexagonal magnet arrays in a uniform applied field. The first and second rows of images show the deposits as the cathode is facing down or up, respectively. In the first column the 500-mT field is aligned with the magnetic axis of a parallel array, in the second column the applied field is zero, and in the third column the 500-mT field is antiparallel to the axis of the parallel array. The third row shows an alternating, downward-facing array in a parallel 500-mT field, zero field, and in antiparallel 500-mT field, respectively. The calculated magnitude of the total field (in Tesla) is plotted in the last row for the hexagonal aligned array in $\pm 500 \mathrm{mT}$ and the hexagonal alternating array in $500 \mathrm{mT}$.

The conclusion is that the inverse patterning is governed by the magnitude of the field acting at the cathode.

\section{Concentration dependence}

Structure appears in the zinc deposits from the $\mathrm{Zn} / \mathrm{Dy}$ bath with an upward-facing cathode at dysprosium concentrations as low as $50 \mu \mathrm{M}$. The structured deposits for $0.1 \mathrm{M} \mathrm{Zn}$ and different concentrations of Dy are illustrated in Fig. 18.
Hexagonal patterns are seen for all dysprosium concentrations in the range $5 \mathrm{mM}$ to $1 \mathrm{M}$, but at the lowest concentrations the pattern becomes indistinct. The 5 and $10 \mathrm{mM}$ solutions of dysprosium are actually diamagnetic $\left(\chi=-6 \times 10^{-6}\right.$ and $-3 \times 10^{-6}$, respectively).

A further effect of changing dysprosium concentration is to modify the conductivity of the solution (Fig. 19). This becomes apparent for concentrations above $10 \mathrm{mM}$, where the transport 


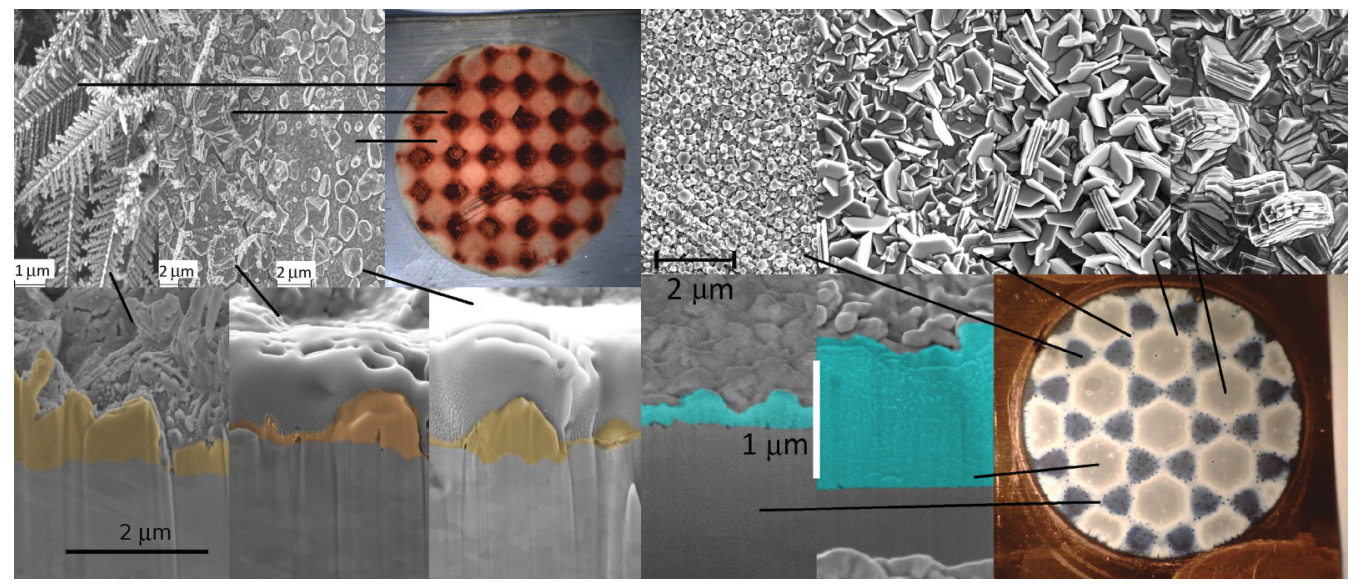

FIG. 11. (Color online) Inversely patterned electrodeposits of $0.1 \mathrm{M} \mathrm{Cu}$ or $\mathrm{Zn}$ in $1 \mathrm{M}$ Dy solution, for an upward-facing cathode with an alternating hexagonal magnet array. Clear patterning is observed for both metals. The images show the surfaces and cross sections of the deposits below and between the magnets.

number is modified by the addition of $\mathrm{Dy}^{3+}$ and $\mathrm{Cl}^{-}$ions. However, the magnet array has no effect on the steady-state current, regardless of dysprosium concentration, as in all cases iR (solution resistance) compensation was employed to ensure the potential drop at the electrode surface remained the same for each concentration, preventing any systematic potentialdependent errors.

A set of experiments were carried out with an electrolyte of $0.1 \mathrm{M} \mathrm{Cu}$, mixed with $3.3 \mathrm{mM}$ Dy. This is a balanced solution, where each component contributes roughly equally to the paramagnetism. In the early stages of deposition, isolated copper crystals with cubic facets about $200 \mathrm{~nm}$ in size, which do not entirely cover the silver substrate, are found scattered across the region between the magnets Fig. 20, and the region below the magnets is covered with the layer of corncob-like copper, similar to that shown in Fig. 4. The copper deposits at this low concentration of Dy are normal, not inverse. A similar effect is seen in the zinc deposits in Fig. 18. Those for Dy concentrations $\leqslant 100 \mathrm{mM}$ are normal (cf. Fig. 4), whereas $1000 \mathrm{mM}$ is inverse (cf. Fig. 15).

The conclusion here is that the inverse patterning operates down to concentrations of dysprosium of order $100 \mathrm{mM}$. At lower concentrations the patterning appears to be normal, even for zinc. This suggests that the phenomenon might be associated with blocking by Dy ${ }^{3+}$ of the reduction of zinc in the double layer, at the cathode surface.

\section{DISCUSSION}

\section{A. Origin of mass transport modulation}

The rich variety of the results obtained on laterally patterned electrodeposits in the previous sections reflects diffusive mass transport, modified by the field gradient force, the Lorentz force, and natural convection. In order to make sense of the
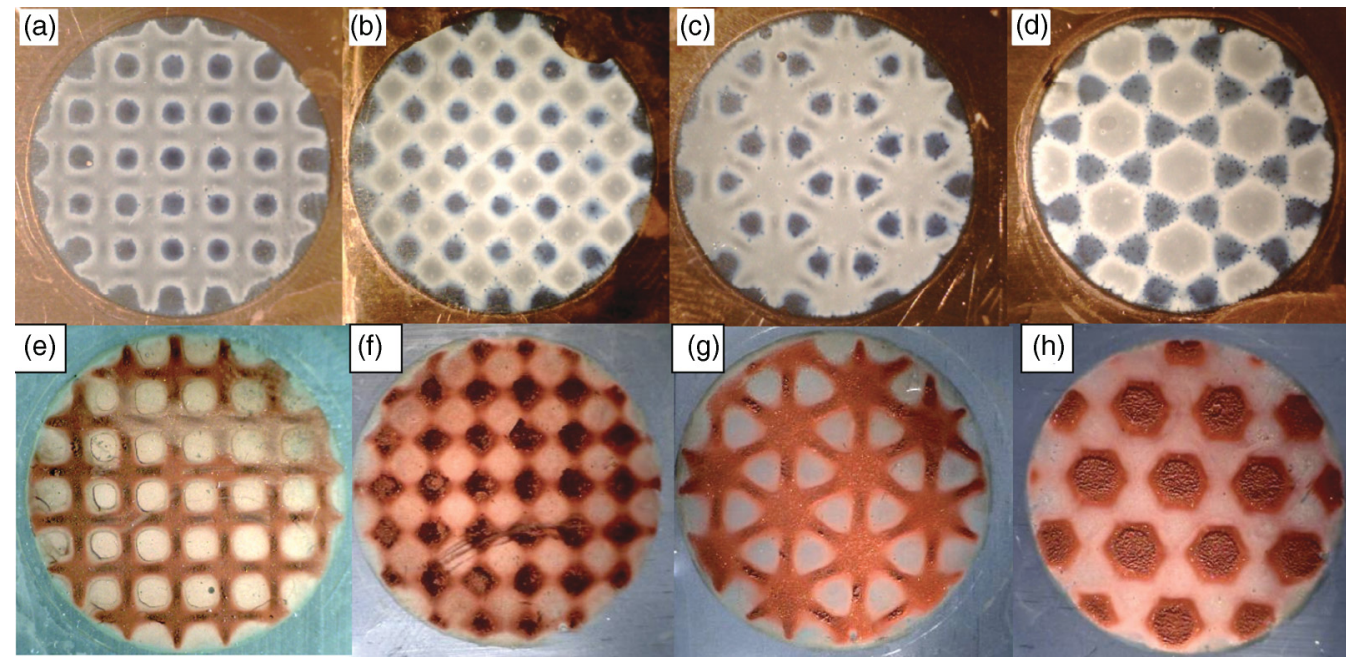

FIG. 12. (Color online) Top row: electrodeposits from $0.1 \mathrm{M} \mathrm{Zn+1} \mathrm{M} \mathrm{Dy} \mathrm{solution} \mathrm{(bath} \mathrm{H}$ ) at $-1.2 \mathrm{~V}$, after $300 \mathrm{~s}$. The magnet arrays are (a) square parallel, (b) square alternating, (c) hexagonal parallel, and (d) hexagonal alternating. Bottom row: electrodeposits from a $0.1 \mathrm{M} \mathrm{Cu}+$ $1 \mathrm{M}$ Dy solution (bath F) at $-5 \mathrm{~mA}$ for $1200 \mathrm{~s}$, for the same sequence of magnet arrays. 


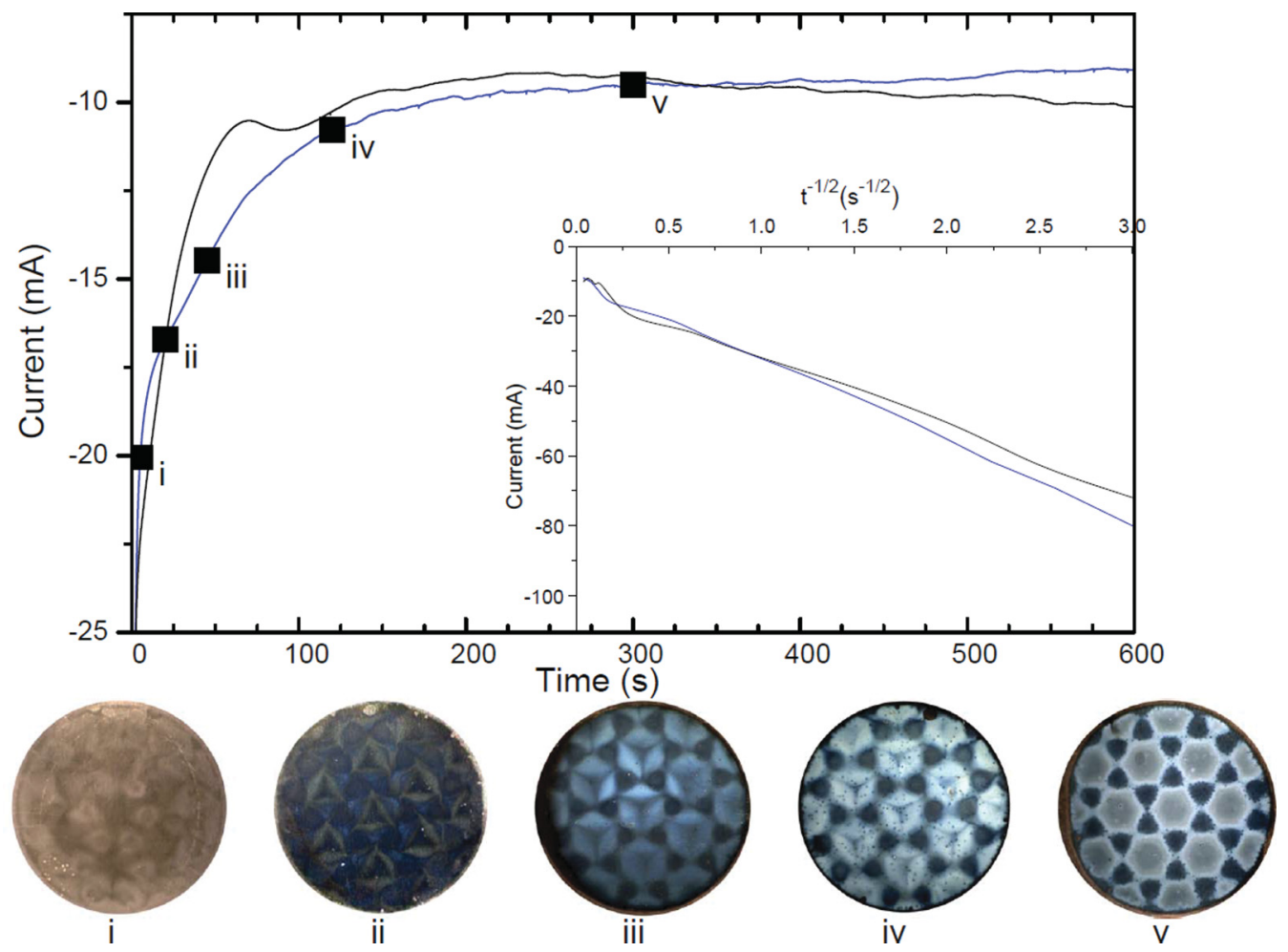

FIG. 13. (Color online) Time-dependence of the current from the $0.1 \mathrm{M} \mathrm{Zn}+1 \mathrm{M}$ Dy bath $\mathrm{H}$ at $-1.2 \mathrm{~V}$ with and without an alternating magnet array. The images show the patterns that have developed after different deposition times for the hexagonal alternating array. The insert is the Cottrell plot used to determine the diffusion constant of zinc.

potentially complex interplay of these effects, we focus on situations in which one effect is dominant.

\section{Lorentz force}

The Lorentz force [Eq. (1)] can often be discounted in our experiments. The limiting current densities we normally

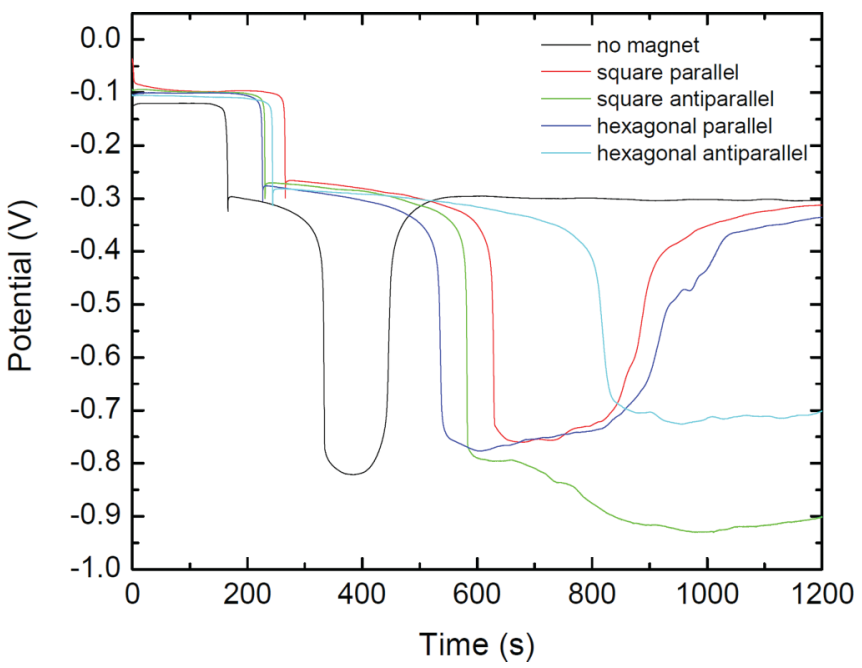

FIG. 14. (Color online) Galvanostatic deposition at a current of $5 \mathrm{~mA}$ from the $0.1 \mathrm{M} \mathrm{Cu}+1 \mathrm{M}$ Dy bath $\mathrm{F}$ at with different magnet configurations; no magnet, square, and hexagonal arrays, parallel and alternating. see with a downward-facing electrode, $10-20 \mathrm{~A} \mathrm{~m}^{-2}$, may be amplified locally by a factor of about 3 when the current is confined by the magnets in the square or hexagonal arrays; the field gradient $\nabla B^{2}=2 B \nabla B$ produced in the vicinity of the cathode by the magnet arrays is of the order $250 \mathrm{~T}^{2} \mathrm{~m}^{-1}$ (Fig. 2), and the $3 d$ susceptibility of the solutions ranges from $2-100 \times 10^{-6}$. Hence, the ratio $R$ [Eq. (5)] ranges from 10 1000; the field gradient force dominates, although the Lorentz force may play a part in the early stages where the transient current densities can be much higher (Figs. 6, 9, and 13).

\section{Natural convection}

Natural convection involves force densities $\Delta \rho \mathrm{g}$ of the order 10-1000 $\mathrm{N} \mathrm{m}^{-3}$, comparable to the field gradient force (Table III). It influences the direct patterns (Fig. 9), but it has little effect on the inverse deposits (Fig. 15), which shows that they are controlled by the large susceptibility of the rare-earth ions. As a baseline to judge the effects of the orientation of the electrochemical cell (convection) and Lorentz force on the deposition current, we ran a set of experiments with $0.1 \mathrm{M} \mathrm{Cu}^{2+}$ solution, bath E. The current densities after $600 \mathrm{~s}$, together with the diffusion layer thickness at that time and the thickness of copper deposited, are included in Table V. The value used for the diffusion constant of $\mathrm{Cu}^{2+}$, $0.50(5) \times 10^{-9} \mathrm{~m}^{2} \mathrm{~s}^{-1}$, was deduced from the Cottrell equation [Eq. (7)], using data for the downward-facing cathode, when the diffusion layer thickness continues to grow with time. The diffusion constant is about half the literature value for 

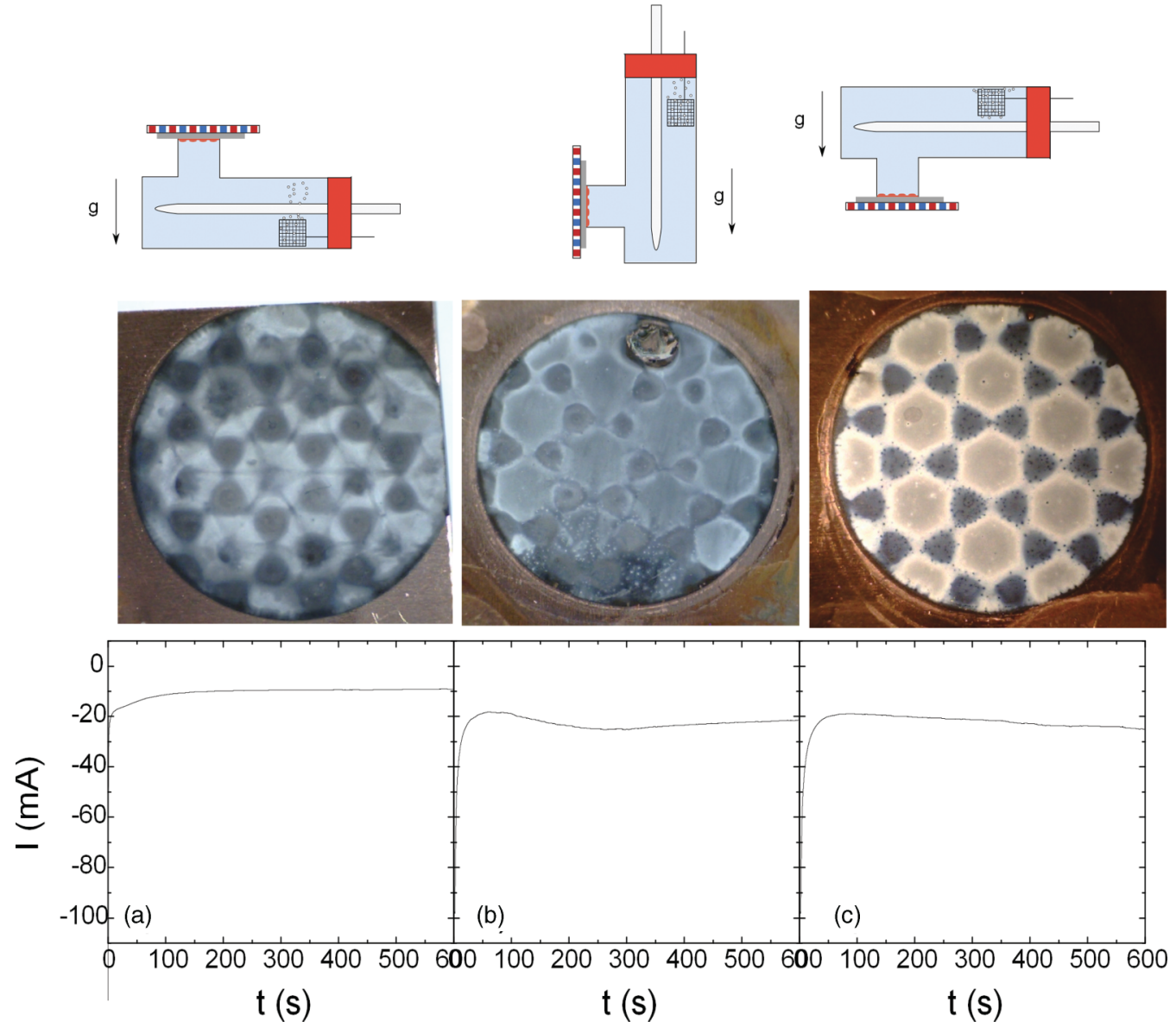

FIG. 15. (Color online) Electrodeposits and current transients obtained with $0.1 \mathrm{M}$ zinc $+1 \mathrm{M}$ dysprosium solution with the cathode facing (a) downwards, (b) vertically, or (c) upwards. The deposits after $600 \mathrm{~s}$ are illustrated.

$0.1 \mathrm{M} \mathrm{Cu}^{2+}$, which may be due to the presence of other ions, especially $\mathrm{Na}^{+}$in the bath. However, in the presence of natural convection, a dynamically stable diffusion layer thickness of

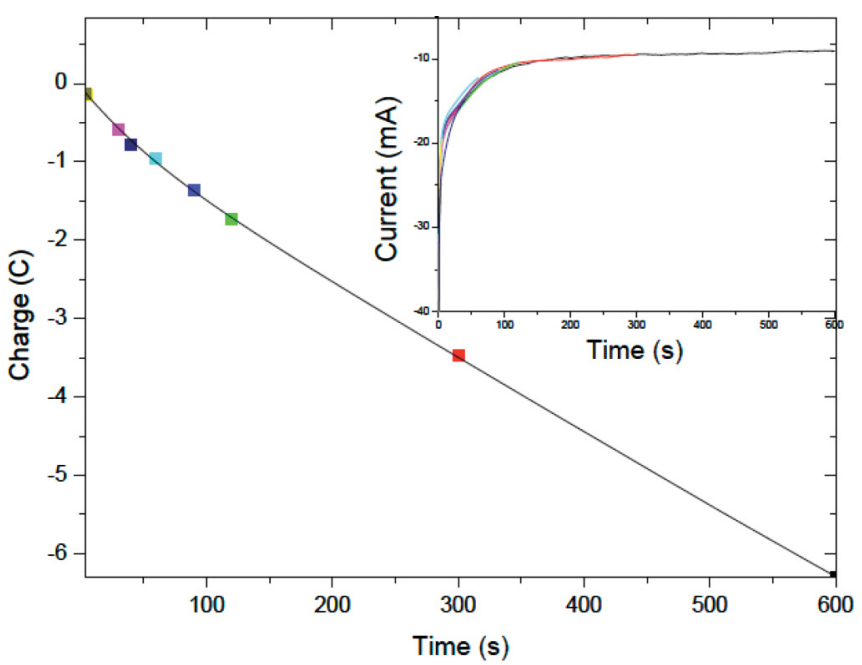

FIG. 16. (Color online) The charge passed as a function of time for the upward-facing configuration in Fig. 15. Ten seconds corresponds to the initial stage of crystallization below and between the magnets. The time points correspond to the images in Fig. 13. about $200 \mu \mathrm{m}$ is established after 20-30 s (see also Figs. 6 and 9 ), and the current is constant thereafter.

\section{Magnetic field gradient force}

When the concentration of magnetic ions in solution is uniform, the energy of each ion is $-(1 / 2)\langle m\rangle B$, where the average moment is given by the Curie law

$$
\langle m\rangle=g^{2} \mu_{B}^{2} \mathrm{~S}(\mathrm{~S}+1) B / 3 \mathrm{k} T .
$$

There will be a field gradient force on each ion of $1 / 2 \mathrm{~g}^{2} \mu_{\mathrm{B}}{ }^{2} \mathrm{~S}(\mathrm{~S}+1) \nabla B^{2} / 3 \mathrm{k} T$. At first sight, the force should produce no observable effect. For $\mathrm{Cu}^{2+}, \mathrm{S}=1 / 2$, the induced moment at $300 \mathrm{~K}$ when $B=1 \mathrm{~T}$ is $2.24 \times 10^{-3} \mu_{\mathrm{B}}$. The energy in $1 \mathrm{~T}$ is $1.1 \times 10^{-26} \mathrm{~J}$, which compares with $\mathrm{k} T=$ $4.1 \times 10^{-21} \mathrm{~J} ; \mu_{\mathrm{B}} / \mathrm{k}=0.67 \mathrm{~K} / \mathrm{T}$. Even for $\mathrm{Dy}^{3+}$, where the induced moment in $1 \mathrm{~T}$ at $300 \mathrm{~K}$ is $8.5 \times 10^{-2} \mu_{\mathrm{B}}$, the energy in $1 \mathrm{~T}$ is still four orders of magnitude less than $\mathrm{k} T$, so the field inhomogeneities will hardly affect the equilibrium concentrations of the ions in solution. The field gradient force on a $\mathrm{Cu}^{2+}$ ion in a field of $1 \mathrm{~T}$ and a gradient of $1 \mathrm{~T} \mathrm{~mm}^{-1}$ is $2 \times 10^{-23} \mathrm{~N}$. Faced with the effective force driving diffusion of the ion $\mathrm{k} T(\nabla c / \mathrm{c}) \approx 4 \times 10^{-17} \mathrm{~N}$, the field gradient force is impotent. The magnetic field gradient cannot drag ions into the vicinity of the cathode. This was checked by filling cuvettes with a $1 \mathrm{M} \mathrm{Er}(\mathrm{NO})_{3}$ solution $\left(\chi_{\mathrm{mol}}=461 \times 10^{-9} \mathrm{~m}^{3} \mathrm{~mol}^{-1}\right)$, 


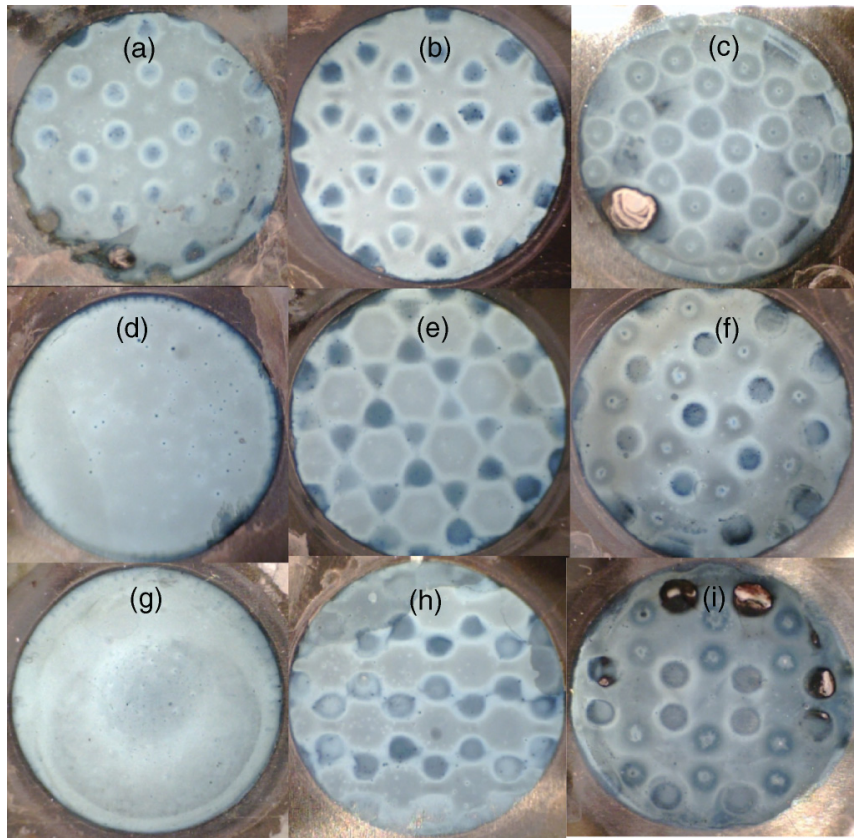

FIG. 17. (Color online) Zinc deposits from the $0.1 \mathrm{M} \mathrm{Zn}+1 \mathrm{M}$ Dy bath in applied fields of $\pm 500 \mathrm{mT}$; hexagonal parallel array in (a) $-500 \mathrm{mT}$, (b) zero field, and (c) $500 \mathrm{mT}$; no magnets, zero field (d); hexagonal alternating array in (e) zero field and (f) $500 \mathrm{mT}$; no magnets in $500 \mathrm{mT}(\mathrm{g})$; hexagonal pairwise array (h) in zero field and (i) in $500 \mathrm{mT}$.

which has a distinct pink color, and placing a magnet outside the cuvette. There was no perceptible change in optical density after several weeks.

However, it is seen in Table III that, when expressed as a body force, the field gradient force can be more than a match for the Lorentz force and comparable to the forces driving natural convection in electrochemical cells. This point is nicely illustrated by the phenomenon of paramagnetic liquid tubes, ${ }^{36,37}$ where a tube of a paramagnetic liquid can be stabilized in water against dissolution by convection, but not
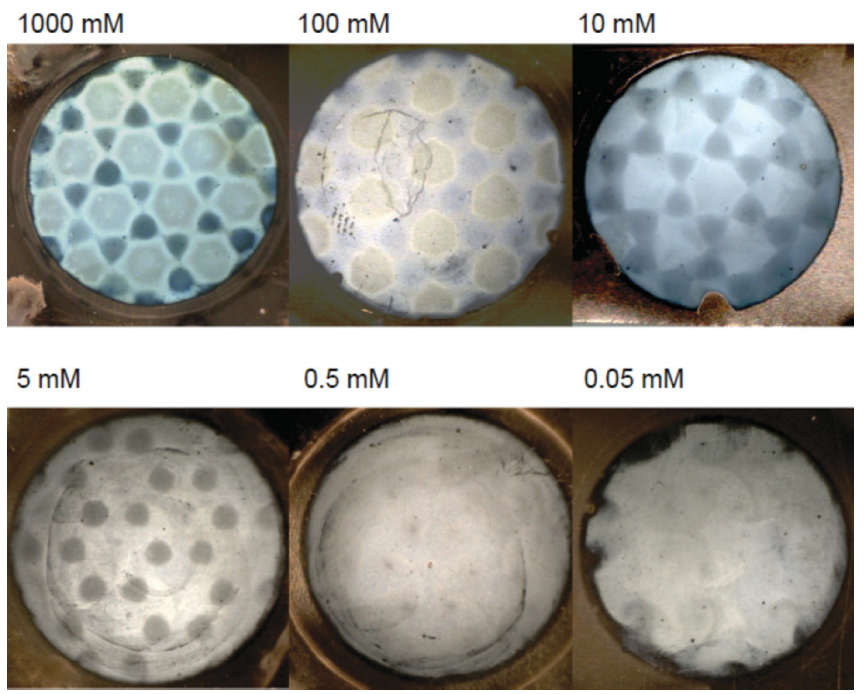

FIG. 18. (Color online) Electrodeposits obtained from a $0.1 \mathrm{M}$ $\mathrm{Zn}+x \mathrm{mM}$ Dy bath for $x=1000,100,10,5,0.5$, and 0.05 .

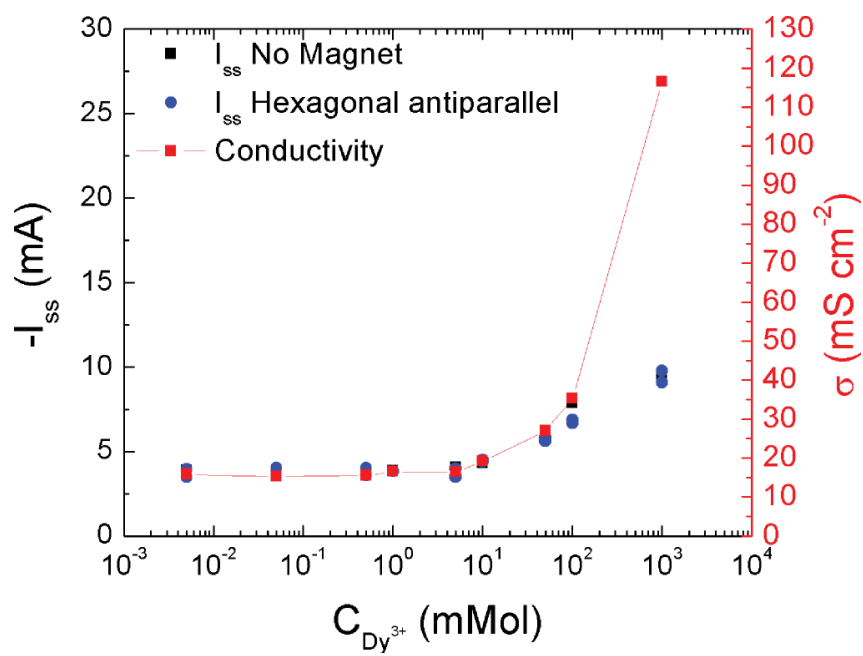

FIG. 19. (Color online) Steady-state currents and conductivity for the electrolytes in Fig. 18, with and without the magnet array.

diffusion, by the field gradient force. The effect of this force is on the kinetics, not the equilibrium of the system. ${ }^{38}$

\section{Magnetic charge model}

The influence of a nonuniform magnetic field reflects not only the magnetic field gradient acting locally on the ions in the paramagnetic electrolyte but also the gradients of susceptibility associated with concentration gradients of the paramagnetic electroactive species. The ideas are best illustrated using the magnetic charge model, which is a convenient way of calculating forces in magnetostatics. Fictitious positive and negative magnetic charges $q_{\mathrm{m}}$ are introduced, which correspond to the North and South poles that feature in science texts for elementary schools. The force on a point charge $q_{\mathrm{m}}$ A m in a field $H$ is $\mu_{0} q_{\mathrm{m}} H$ newtons. Given a magnetization distribution $\boldsymbol{M}(\boldsymbol{r})$, the magnetic charge appears wherever there is a nonuniform spatial distribution of magnetization. At a sharp interface with a normal unit vector $\mathbf{e}_{\mathrm{n}}$, the surface charge density is $\sigma_{\mathrm{m}} \mathrm{A} \mathrm{m}^{-1}$, where $\sigma_{\mathrm{m}}=\boldsymbol{M}$. $\mathbf{e}_{\mathrm{n}}$. More generally,

TABLE V. Current densities, diffusion layer thickness, and thickness of copper deposited after $600 \mathrm{~s}$ for a $0.1 \mathrm{M} \mathrm{Cu}^{2+}$ bath. The applied field is $500 \mathrm{mT}$. A right-handed coordinate system is used with $z$ vertical.

\begin{tabular}{lcccc}
\hline \hline $\begin{array}{l}\text { Field } \\
\mathrm{T}\end{array}$ & $\begin{array}{c}\text { Electrode } \\
\text { normal }\end{array}$ & $\begin{array}{c}j \\
\mathrm{~A} \mathrm{~m}^{-2}\end{array}$ & $\begin{array}{c}\delta \\
\mu \mathrm{m}\end{array}$ & $\begin{array}{c}t_{\mathrm{Cu}} \\
\mu \mathrm{m}\end{array}$ \\
\hline 0 & $z$ (up) & 56 & 179 & 1.25 \\
0 & $x$ & 44 & 227 & 0.96 \\
0 & $-z$ (down) & 15 & 667 & 0.53 \\
$z 0.5$ & $z$ (up) & 51 & 196 & 1.14 \\
$z 0.5$ & $x$ & 43 & 233 & 0.94 \\
$z 0.5$ & $-z$ (down) & 7 & 1430 & 0.32 \\
$y 0.5$ & $x$ & 38 & 263 & 0.86 \\
$-y 0.5$ & $x$ & 43 & 233 & 0.93 \\
$x 0.5$ & $x$ & 33 & 303 & 0.71 \\
$x 0.5$ & $-z$ (down) & 9 & 1072 & 0.50 \\
$x 0.5$ & $y$ & 45 & 214 & 1.06 \\
$x 0.5$ & $z$ (up) & 64 & 151 & 1.44 \\
\hline \hline
\end{tabular}


TABLE VI. Estimated diffusion layer thickness from $\mathrm{Cu}$ deposition from bath $\mathrm{E}$ using FIB cross-section data with current transients, compared to the expected modulation from the magnetic pressure model for (a) square parallel, (b) square alternating, (c) hexagonal parallel, and (d) hexagonal alternating arrays.

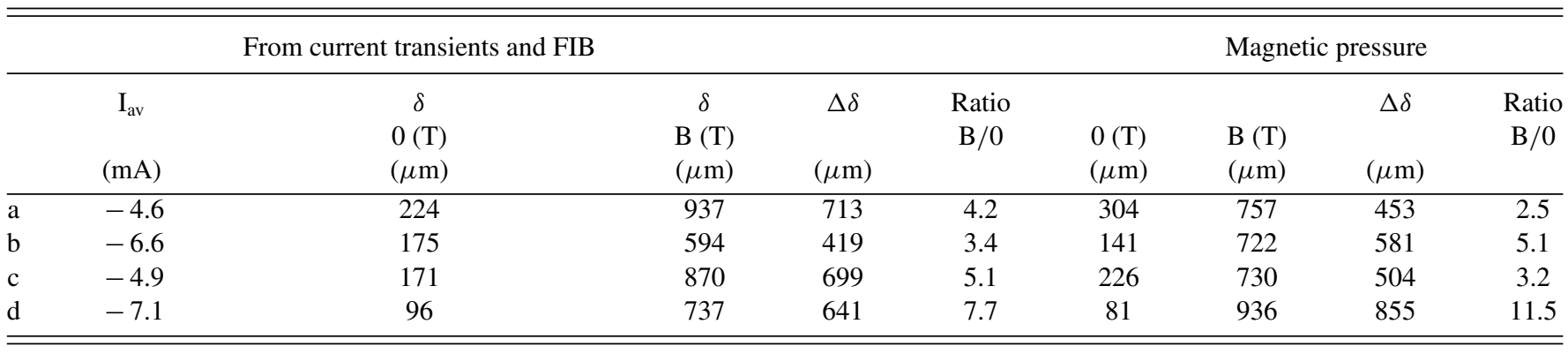

when $\boldsymbol{M}$ is nonuniform, there is a volume charge density $\rho_{\mathrm{m}}=$ $-\nabla \cdot \boldsymbol{M ~ A ~ m}{ }^{-2} .^{28}$

Several different cases for a confined electrolyte are compared in Fig. 21. The field is either uniform or nonuniform, and the concentration gradient at the interface is either sharp or diffuse.

Case 1: A sharp interface in a uniform field. In the first case, sharp interfaces in a uniform field, a surface density of magnetic charge is induced along the top and bottom interfaces. The induced magnetization is $\boldsymbol{M}=\chi \boldsymbol{H}$, so a pressure

$$
P_{\mathrm{m}} \approx\left(1 / 2 \mu_{0}\right) c \chi_{\mathrm{mol}} B^{2}
$$

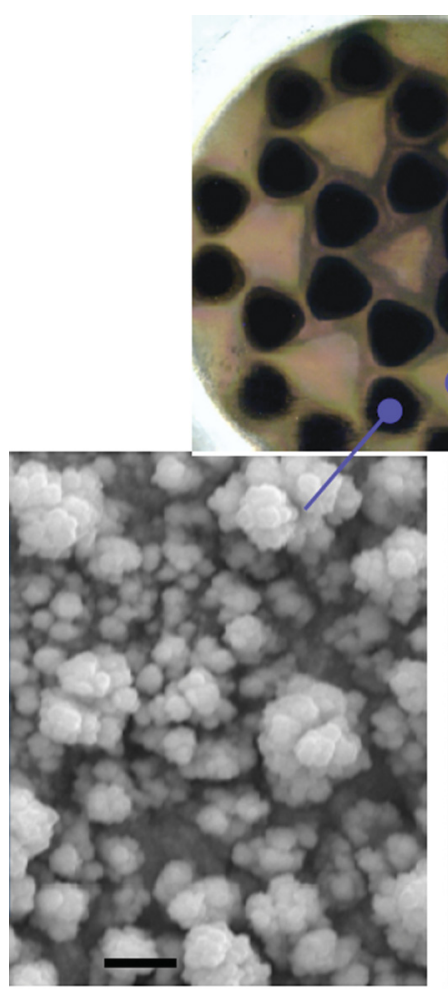

(a)
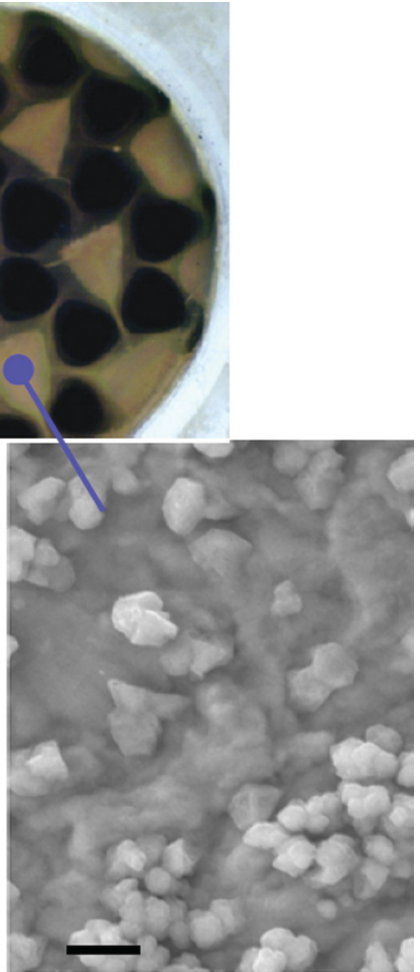

(b)
FIG. 20. (Color online) Scanning electron micrographs of the early stages of copper deposition from a balanced electrolyte, $0.1 \mathrm{M}$ $\mathrm{Cu}+3.3 \mathrm{mM}$ Dy. (a) shows the deposit of corncob-like copper about $2 \mu \mathrm{m}$ thick, which forms below the magnets, and (b) shows the sprinkling of $\sim 200$-nm copper crystallites on the silver surface. acts normal to the two interfaces. This stress, or magnetic pressure is of the order $0.5 \mathrm{~Pa}$ for $1 \mathrm{M} \mathrm{Cu}^{2+}$ solution in $300 \mathrm{mT}$, but it has no effect on the electrolyte because it is balanced by the normal reaction from the walls of the container. We can ignore it.

Case 2: A diffuse interface in a uniform field. Next we consider a diffuse interface in a uniform field, where $\mathrm{c}(z)=$ $\mathrm{c}_{0} z / \delta$ for $z<\delta$ and $\mathrm{c}(z)=\mathrm{c}_{0}$ for $z>\delta$. The volume charge density in the diffusion layer is uniform $\rho_{\mathrm{m}}=d M / d z=$ $\mathrm{c}_{0} \chi H / \delta$, so the total force per unit area is $\mathrm{c}_{0} \chi_{\mathrm{mol}} B^{2} / \mu_{0}$, as before. There is a compressive stress on the diffusion layer, but this also has no effect provided the field is uniform. It does not matter whether the interface is sharp or diffuse; there is no net force on a paramagnetic medium in a uniform field.

Case 3: Uniform concentration in a nonuniform field. Even when the field is nonuniform, there is no unbalanced force provided the electrolyte is contained, and the concentration and susceptibility are uniform. The $z$ component of the field of a magnetic point dipole falls off as $z^{-3}$, so when $z / \delta>10$, the field has fallen to a small value. There is essentially no normal component of the field at the lower cell wall [Figs. 1(a) and 21(c)], and so there is no magnetic charge there. The magnetic force acts only at the upper interface, but it is balanced by the normal reaction from the wall. There are pressure variations along the wall, but they have no effect in the closed system. Furthermore there is no force on the magnetic charge distribution induced in the volume of the electrolyte. The induced magnetization $\boldsymbol{M}(\boldsymbol{r})=\mathrm{c}_{0} \chi \boldsymbol{B}(\boldsymbol{r}) / \mu_{0}$. But the volume magnetic charge density $\rho_{\mathrm{m}}=-\nabla \cdot \boldsymbol{M}=$ 0 , because $\nabla \cdot \boldsymbol{B}=0$. In other words, there is no magnetic force on any part of the electrolyte where the concentration is uniform.

Case 4: A free upper surface in a nonuniform field. Consider next the situation in which the upper surface is free. It becomes an equipotential surface responding to the magnetic pressure [Eq. (9)]. In pure water, this is known as the "Moses effect." Variations in the depth of water $\Delta h$ reflect $P_{\mathrm{m}}$ according to Archimedes' principle

$$
\Delta h \rho g=\left(1 / 2 \mu_{0}\right) \chi B^{2},
$$

where the surface of water is depressed by $0.36 \mathrm{~mm}$ in a nonuniform field of $1 \mathrm{~T}$ because of its diamagnetic susceptibility $\chi=-9 \times 10^{-6}$. Since the effect is quadratic in field, a more impressive Moses effect is observed in fields $>10 \mathrm{~T}^{39}$ Much larger effects are found for strongly 
(a)

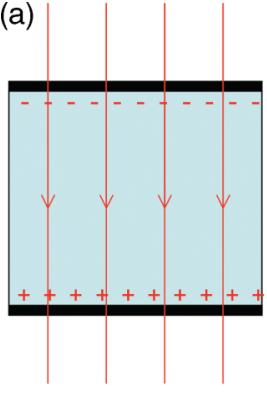

(d)

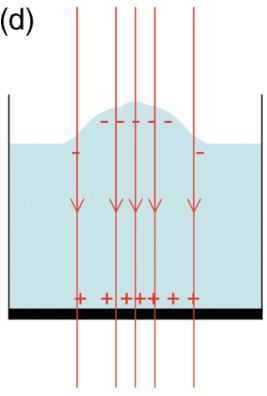

(b)

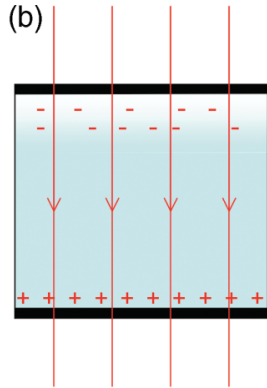

(e)

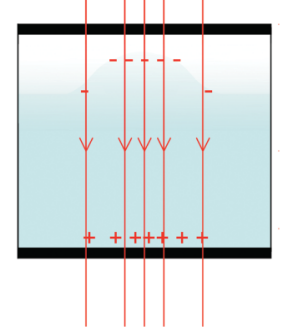

(c)

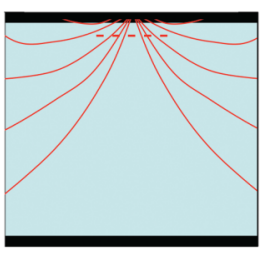

(f)

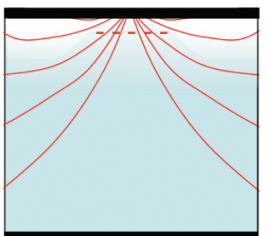

FIG. 21. (Color online) Three cases in which the magnetic field has no effect on the paramagnetic interface. (a) Uniform field, no free surface; (b) uniform field, concentration gradient; (c) nonuniform field, no free surface, and three cases in which there is a redistribution of liquid in the field: (d) nonuniform field, free surface; (e) and (f) nonuniform field, concentration gradient.

paramagnetic solutions; the surface of $1 \mathrm{M} \mathrm{Dy}^{3+}$ for example $\left(\chi=585 \times 10^{-6}\right)$ will be raised by $20 \mathrm{~mm}$ in $1 \mathrm{~T}$, and it would be raised by $2 \mathrm{~m}$ in $10 \mathrm{~T}$, if funding could be found for such an experiment. Likewise, the height of interfaces of immiscible paramagnetic and diamagnetic fluids is readily modulated by an in-plane nonuniform magnetic field, i.e., parallel to the liquid-liquid interface. ${ }^{40}$ An extreme example is the free surface of a ferrofluid $(\chi \approx 1)$, which adopts a characteristic peaked structure as it endeavors to minimize its energy in the sum of the applied and demagnetizing fields. ${ }^{41}$

Cases 5 and 6: Concentration gradient in a nonuniform field. The fifth and sixth panels in Fig. 21 show a nonuniform field acting on a diffuse interface. This corresponds to the situations we have been studying. The pressure variations along the interface can now be compensated by modifying the concentration profile. For example, if the vertical component of the field varies from zero to $B_{\mathrm{z}}$, the pressure change on the paramagnetic electrolyte is compensated by modulating the thickness of the diffuse layer at the interface by an amount $\Delta \delta$ so that

$$
\Delta \delta \Delta \rho g=\left(1 / 2 \mu_{0}\right) \chi_{\mathrm{mol}} c_{0} B_{z}^{2}
$$

In the case of $0.1 \mathrm{M} \mathrm{Cu}^{2+}$ and $B_{\mathrm{z}}=0.3 \mathrm{~T}, \Delta \rho=15 \mathrm{~kg} \mathrm{~m}^{-3}$, and the modulation of the diffusion layer thickness is $0.7 \mathrm{~mm}$. This is the same order of magnitude as the thickness of the layer itself (Table V), hence, the influence of a nonuniform magnetic field on the thickness of the electrodeposit. This can be extended by including the total pressure change due to the magnitude of the magnetic field, $|\boldsymbol{B}|$, and is in agreement with total energy density approach of the Moses effect, ${ }^{40}$ where

$$
\Delta \delta \Delta \rho g=\left(1 / 2 \mu_{0}\right) \chi_{\mathrm{mol}} c_{0} B^{2} .
$$

\section{Vorticity force analysis}

It should be noted that the magnetic field gradients necessary to produce measurable effects are not huge. It is enough for the nonuniform field to vary on a length scale greater than that of the concentration variations. We can see this more formally by looking at the effects of the field gradient force and the Lorentz force on hydrodynamic flow. ${ }^{30,42}$ Vorticity in hydrodynamics is induced by the curl of the body force. Any force field for which $\nabla \times \boldsymbol{F}=0$ is conservative, meaning it cannot induce convection, although it can modify or bifurcate existing convective flows. This is another way of expressing two mathematically equivalent statements: (i) the force is path independent, i.e., the force integral over a closed loop, or the net work done is zero, and (ii) the force can be written as the gradient of a potential. When $\nabla \times \boldsymbol{F} \neq 0$, the force field is nonconservative, and it can induce convective motion in the fluid. It is possible to use this analysis to study the properties of $\boldsymbol{F}_{\mathrm{L}}$; however, it is questionable whether or not it makes physical sense to consider the curl of the Lorentz force. This is because the curl acts on a vector field, but the Lorentz force is not a simple vector field since it is also a function of velocity. Even so, if we take the curl of $\boldsymbol{F}_{\mathrm{L}}$ and use the vector identity $\nabla \times \boldsymbol{j} \times \boldsymbol{B}=(\nabla \cdot \boldsymbol{B}) \boldsymbol{j}-(\nabla \cdot \boldsymbol{j}) \boldsymbol{B}+(\boldsymbol{B} \cdot \nabla) \boldsymbol{j}-$ $(\boldsymbol{j} \cdot \nabla) \boldsymbol{B}$, we see that

$$
\nabla \times \boldsymbol{F}_{L}=(\boldsymbol{B} \cdot \nabla) \boldsymbol{j}-(\boldsymbol{j} \cdot \nabla) \boldsymbol{B}
$$

since the divergence of both $\boldsymbol{B}$ and $\boldsymbol{j}$ are zero. In a configuration where $\boldsymbol{B}=B_{x}$ and $\boldsymbol{j}=j_{z}, \nabla \times \boldsymbol{F}_{\mathrm{L}}=B_{x} d j_{z} / d x-$ $j_{z} d B_{x} / d z$. This is zero if both the field and current density are uniform. The latter can never be true in any electrochemical cell with finite electrodes, and vortices are known to appear close to the edge of the electrode. ${ }^{43}$

A further complication is that while the Lorentz force might approximately satisfy the zero curl condition in a very large cell and satisfy condition (i), considering that the work done by a magnetic field on a charged particle is always zero due to the force being perpendicular to the direction of motion, it does not satisfy condition (ii). It is not possible in general to describe the Lorentz force solely in terms of a scalar potential. Therefore, the Lorentz force should be classified as nonconservative. This is in line with the observation that the Lorentz force can indeed induce convective flow, and at high current densities, turbulence.

A major source of vorticity in an electrochemical cell is the boundary between the electrolyte and the cell walls. At large Hartmann numbers, an exponential hydrodynamic boundary (Hartmann) layer is formed close to the fluid-wall interface, ${ }^{42}$ thus pushing the vorticity toward the boundary. An example of this is the Aogaki cell. ${ }^{1,2}$ Poiseuille flow through the duct is observed at low Hartmann numbers, while at large Hartmann numbers compact boundary layers form at each wall, leaving a uniform flow profile in the center. In the present cell, $\mathrm{Ha} \approx 1$ (Table I).

Turning now to the field gradient force and taking the curl of $\boldsymbol{F}_{\nabla \mathrm{B}}$, it is possible to distinguish two regions 
where the influence of the field gradient force is different. $\left.\nabla \times \boldsymbol{F}_{\nabla \mathrm{B}}=\nabla \times 1 / 2 \mu_{0}\right) \chi_{\mathrm{m}} c \nabla B^{2}$, which can be written as $\left(1 / 2 \mu_{0}\right) \chi_{\mathrm{m}}\left[\left(\nabla c \times \nabla B^{2}+c \nabla \times B^{2}\right]\right.$. Since $\nabla \times \nabla \psi=0$ and relating the magnetic field to a scalar potential, which is possible in the absence of conduction currents, we have

$$
\nabla \times \boldsymbol{F}_{\nabla B}=\left(1 / 2 \mu_{0}\right) \chi_{\mathrm{m}}\left(\nabla c \times \nabla B^{2}\right) .
$$

The inclusion of an electric current does not invalidate this expression, as the typical currents used here $(-20 \mathrm{~mA}$ to $-2 \mathrm{~mA}$ ) create a negligible magnetic field $\sim 10^{-8} \mathrm{~T}$. In the bulk solution, where there is no concentration gradient, the force is conservative. However, close to an electrode interface where a concentration gradient exists in the diffusion layer, the field gradient force is able to induce local convective flows. The condition is that the concentration gradient and the field gradient must have orthogonal components.

\section{B. Normal patterns}

We therefore expect to explain the normal structuring in terms of the magnetic field acting in the diffusion layer of the electrolyte. We can safely ignore any direct influence of the field gradient force on diffusion. However, the field gradient force [Eq. (4)] is of the order $500 \mathrm{~N} \mathrm{~m}^{-3}$ when $B=300 \mathrm{mT}$, $\chi=10 \times 10^{-6}$, and $\nabla B=200 \mathrm{~T} \mathrm{~m}^{-1}$, which is comparable to the force density driving convection, $\Delta \rho \mathrm{g}$. As a result, we expect to encounter modifications of the convective regime due to the field gradient.

The simplest experimental conditions are with the downward-facing cathode, which minimizes convection. Not only is metal deposited at the cathode, there is also the backflow of water, which is involved in convection in the steady state. The water shed at the cathode tends to be stabilized gravitationally just below the cathode surface, but it is recycled by the convective processes, which lead eventually to dynamic equilibrium of the diffusion layer. This occurs after about $1000 \mathrm{~s}$ for the downward-facing cathode, but it takes only about $20 \mathrm{~s}$ when natural convection is active (Fig. 9). The relation between current density and rate of deposition of copper, assuming $100 \%$ current efficiency, is

$$
j=2000 \mathrm{~F}\left(d t_{\mathrm{Cu}} / d t\right) \rho_{\mathrm{Cu}} / \mathcal{M}_{\mathrm{Cu}},
$$

where $\rho_{\mathrm{Cu}}$ and $\mathcal{M}_{\mathrm{Cu}}$ are the density and atomic weight of copper. Equivalent expressions apply for other metals. When $j$ is uniform, a current of $10 \mathrm{~mA}$ will deposit $1.25 \mathrm{~nm}$ of copper per second on the $2.8 \mathrm{~cm}^{2}$ electrode. Since the volume of water in the hydration sphere is about 40 times the volume of copper deposited, about $60 \mathrm{~nm}$ of water produced every second is recycled back into the bath. From diffusion considerations [Eq. (6)], we see that not much accumulation of water at the cathode is expected. The self-diffusion constant of water is $2.2 \times 10^{-9} \mathrm{~m}^{2} \mathrm{~s}^{-1}$. After $1000 \mathrm{~s}$, the $60 \mu \mathrm{m}$ of water produced will have largely diffused away. Diffusion is the only process involved in the absence of the magnetic field for the downward-facing electrode, but when a laterally fluctuating field is present, convective flows are induced in the diffusion layer by the field gradient force, Eq. (14) (Fig. 6).

The downward-facing cathode is intended to mitigate natural convection due to density differences in the electrolyte. The copper deposits with the downward-facing cathode are most clearly defined for the $0.1 \mathrm{M}$ copper bath. There the limiting current is $2 \mathrm{~mA}$ after $1200 \mathrm{~s}$, Fig. 6, corresponding to $j=7 \mathrm{~A} \mathrm{~m}^{-2}$, and the copper diffusion layer thickness deduced from Eq. (2) is $1340 \mu \mathrm{m}$. In fact the diffusion layer thickness will be modulated by the magnetic field so that it is thinner where the deposition rate is higher. ${ }^{24}$ The cross-section data in Fig. 4(b) show that the deposition thickness varies by a factor of 4 across the hexagonal magnet array. This can be simply related to the modulation in diffusion layer thickness given by Eq. (10). If we assume a steady state and use the suffixes 1 and 2 to represent the positions below and between the magnets, then from Eq. (2), $j_{1} / j_{2}=\delta_{2} / \delta_{1}$. The magnets occupy $28 \%$ of the area of the hexagonal arrays, so $j=(0.28) j_{1}+(0.72) j_{2}$. A rough description of the deposition process can be given in terms of the time average current. This is $5 \mathrm{~mA}$ over the first $600 \mathrm{~s}$, based on the charge passed $(3 \mathrm{C})$; hence, $j_{\mathrm{av}}=18 \mathrm{~A}$ $\mathrm{m}^{-2}$. The ratio $j_{1 \mathrm{av}} / j_{2 \mathrm{av}}=4$, so $j_{1 \mathrm{av}}=40 \mathrm{~A} \mathrm{~m}^{-2}$ and $j_{2 \mathrm{av}}=$ $10 \mathrm{~A} \mathrm{~m}^{-2}$. The corresponding values of the diffusion layer thicknesses deduced from Eq. (2) with $D=0.5 \times 10^{-9} \mathrm{~m}^{2} \mathrm{~s}^{-1}$ are $\delta_{1}=984 \mu \mathrm{m}$ and $\delta_{2}=241 \mu \mathrm{m}$. Equation (11), $\left(\delta_{2}-\right.$

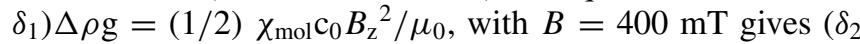
$\left.-\delta_{1}\right)=684 \mu \mathrm{m}$. The results of a fuller analysis are presented in Table VI, where FIB cross-section data for each array were combined with the measured current transients to compare the effective diffusion layer modulation to that expected from the magnetic pressure model. For all arrays, the model can give a semiquantitative account of the deposit thickness ranging from 100 to $1000 \mathrm{~nm}$ and diffusion layer thicknesses ranging from 100 to $1000 \mu \mathrm{m}$. Magnetic pressure, therefore, accounts for the modulation of the deposit thickness by the magnetic field.

The agreement is better than could reasonably be expected, in view of the approximations made and the experimental uncertainties in the deposit thickness ratio (measured at only two points) and the diffusion constant. The magnetic field is not actually constant as the diffusion layer evolves with time, as the field falls off rapidly with $z$. The value at the cathode surface is $300 \mathrm{mT}$, but at $500 \mu \mathrm{m}$ from the surface, it has fallen to $160 \mathrm{mT}$ (Fig. 22). The contrast tends to disappear at long times for this reason, but also as the diffusion layer thickness becomes much greater than the fixed difference $\left(\delta_{2}-\delta_{1}\right)$ given by Eq. (12), the ratio of the currents $\delta_{2} / \delta_{1}$ becomes small. This is clearly seen in Fig. 23, where the effects of Eq. (12) are included in the growth of the diffusion layer with time. In agreement with the experimental data (cf. Fig. 6), the greatest differences are at intermediate times when the diffusion layer modulation is largest, while the limiting currents for the downward-facing cathode tend to the same value at long times regardless of the magnet array as the modulation is smeared out after $10 \mathrm{~min}$ as the diffusion layer grows.

Also of note is that, on average, the diffusion layer stays thinner for longer below the alternating arrays compared to the parallel arrays. This fits with the measured current densities at intermediate times being considerably greater for the alternating arrays. This was shown in Fig. 8 for a square array, but it has been observed systematically with other arrays as well.

The neglect of lateral diffusion and flow also becomes less justified as time runs on, and the thickness of the diffusion layers become comparable to the separation of the magnets. Lateral diffusion will deform the diffusion layer profile and may lead to more angular dot shapes. Furthermore, there is 


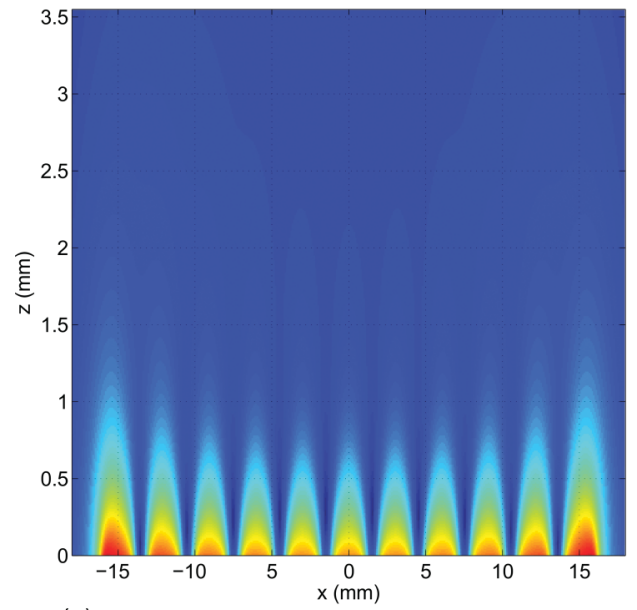

(a)

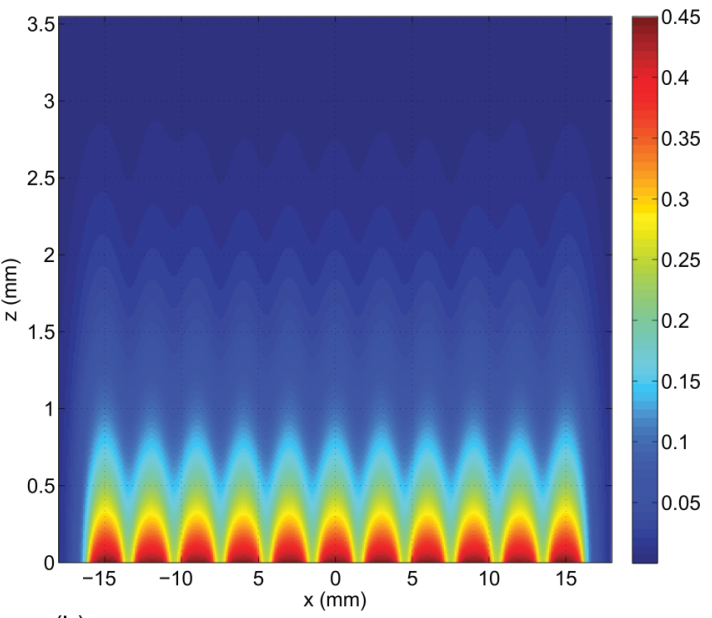

(b)

FIG. 22. (Color online) Vertical profiles of $|\boldsymbol{B}|$ along the central $x$ axis for (a) a square parallel array and (b) a square alternating array.

little contrast in the initial stage of electrodeposition, because the thicknesses of the two diffusion layers have not yet reached the difference given by Eq. (12). These short- and long-time predictions of the magnetic pressure model are in accord with experimental observations on copper. There is an optimum, intermediate time when the patterns are best developed. A detailed time-dependent three-dimensional diffusion calculation, taking full account of the spatial variation of the magnetic field is feasible, but probably unjustified by the present data.

Since the scale of the deposits available is limited by the diffusion layer thickness and modulation thickness, there is a lower limit on the smallest possible deposited dimensions. We have been able to deposit structured arrays on lithographically patterned magnetic dots as small as $80 \mu \mathrm{m}$ in size, but it is unlikely that this method could be used below $10 \mu \mathrm{m}$.

When hydrogen is evolved at a downward-facing electrode at high overpotential, the gas bubbles tend to congregate in the regions of low field, between the magnets, thereby enhancing the normal patterns.

Another important aspect of our results is the difference in texture when the deposits are produced in the magnetic field. Such effects have been quite extensively documented in the literature, mainly for copper, ${ }^{8,17,44}$ and it has been suggested that they are somehow associated with convection generated by the Lorentz force. ${ }^{45,46}$ Aogaki $^{47}$ has proposed that the field effect on two-dimensional nucleation is related to spontaneous concentration fluctuations in the double layer, and the effect on three-dimensional nucleation depends on fluctuations in the diffusion layer. One aspect of the magnetic-field-induced texture is simply related to the deposition rate. Copper deposits tend to become increasingly dendritic as the diffusion-limited current density is approached, ${ }^{48}$ so the normal copper deposits are rougher in the magnetic field, where the current density is higher. At constant current density, however, the deposits in the field tend to be smoother.

The normal magnetic structuring effect, for copper at least, is disrupted for an upward-facing or vertical electrode. There, the water shed at the electrode leads to natural convection, which tends to destroy any structure due to magnetic pressure in the diffusion layer, as shown in Fig. 9.

\section{Inverse patterns}

The inverse deposits obtained when electroactive copper or zinc was mixed with a dysprosium salt, which has a high paramagnetic susceptibility $\chi_{\mathrm{mol}}=594 \times 10^{-9} \mathrm{~m}^{3} \mathrm{~mol}^{-1}$ but is not electroactive at the deposition potentials used, are mostly obtained in a different regime to the direct deposits. The $\mathrm{Zn}$ and $\mathrm{Cu}$ solutions and also pure water now behave as if they have a huge diamagnetic susceptibility $\chi \approx-590 \times 10^{-6}$ relative to the $1 \mathrm{M}$ Dy background. This effect has been used to levitate various solids and liquids in a modest field gradient of the order $30 \mathrm{~T}^{2} \mathrm{~m}^{-1}, 33,39,49$ compared with the value $1000 \mathrm{~T}^{2} \mathrm{~m}^{-1}$ usually needed to levitate water, frogs, or strawberries in air. ${ }^{50-52}$ In the inverse deposits, the antidots below the magnets are thinly plated, and the deposit is thick in regions between the magnets, where the field is smallest.

The effective magnetic pressure acting on the electroactive solution is $1 / 2 \chi B^{2} / \mu_{0}$, which is now $\sim-20 \mathrm{~Pa}$ in $300 \mathrm{mT}$. Taking a density difference of $100 \mathrm{~kg} \mathrm{~m}^{-3}$ for the electrolyte gives a value of $\Delta \delta$ from Eq. (11) of the order of a centimeter, which could never be reached in our experimental arrangement. It is impossible to structure the diffusion layer on a length scale that is much greater than the separation between the magnets. The magnetic pressure model in its simple form breaks down.

Although the magnetic pressure is much greater than before, and of opposite sign, we have seen from the discussion of Fig. 21 and Eq. (13) that it can have no effect unless a concentration gradient is simultaneously present. That concentration gradient is established in the first few seconds of electrodeposition, as shown by the time sequence in Fig. 13. Convection then builds up and moves the electroactive electrolyte with the large effective diamagnetic susceptibility into the low-field regions to create the lateral concentration gradient, which is reflected in the patterned electrodeposits.

The patterns reflect the field distribution, being distinctly different for parallel and alternating arrays, see for example Fig. 12. There are also features such as the lines and Mercedes stars, which are evident in Figs. 13 and 15, which do not reflect any feature in the field maps (Fig. 2). These features are 

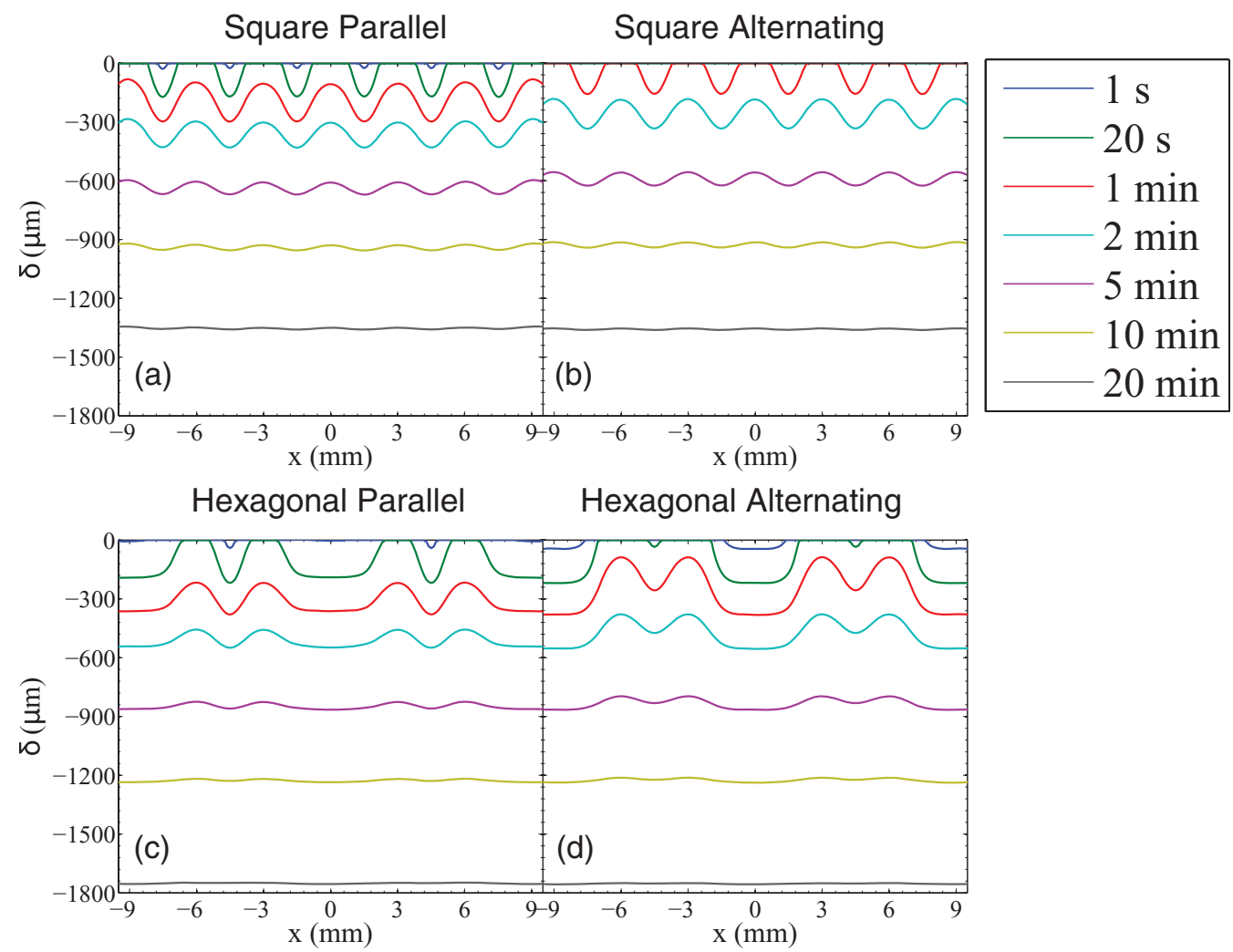

FIG. 23. (Color online) Evolution of the diffusion layer along the central $x$ axis over time for $0.1 \mathrm{M} \mathrm{CuSO}_{4}$ using the magnetic pressure model below: (a) a square parallel array, (b) a square alternating array, (c) a hexagonal parallel, and (d) a hexagonal alternating array.

evidence that Lorentz forces are having a secondary influence on the convective flows, since they appear particularly when there is a significant in-plane component to the field.

Magnetic pressure certainly keeps the $\mathrm{Dy}^{3+}$ ions in the close vicinity of the magnets at high concentrations. However, it is seen in Fig. 18 that the magnetic patterning persists at concentrations as low as $5 \mathrm{mM}$, where the paramagnetism of the dysprosium is overcome by the diamagnetism of water. This interesting observation suggests that $\mathrm{Dy}^{3+}$ ions accumulated in the vicinity of the magnets are blocking cation sites in the double layer, thereby rendering them inaccessible to $\mathrm{Cu}^{2+}$ or $\mathrm{Zn}^{2+}$ ions and impeding their reduction to metal. Charge neutrality induces an increased concentration in the inert $\mathrm{Dy}^{3+}$, along with $\mathrm{H}^{+}$, originating in the double layer, and diffusing out into the diffusion layer.

Finally, it must be emphasized that there are drastic differences in texture of the deposits in different places. For copper, the growth rate in the areas between the magnets is very high; the current density there is of order $150 \mathrm{~A} \mathrm{~m}^{-2}$. Many of the deposits with $1 \mathrm{M}$ Dy are grown on an upward-facing cathode, since natural convection does not have much effect and it is more convenient to make the measurements this way. The dendrite forests reflect the rapid copper growth rate, and the deposits appear rough and brown between the magnets and bright and smooth between them, where the deposit is thin (Fig. 11). The texture effects for zinc are different; the end-on growth of zinc crystallites below the magnets makes these areas appear darker than the thicker, well-aligned areas between them.

\section{CONCLUSIONS}

Magnetic fields produced by single magnets and magnet arrays can be used to structure electrodeposits of both paramagnetic and diamagnetic ions, in patterns reflecting the magnetic field near the cathode in ways that are often strange and unpredictable. There are lateral variations of both the thickness and the texture of the electrodeposits, which have to be characterized before jumping to conclusions based solely on the optical images, which are sensitive to the mode of illumination.

Magnetic pressure is a very useful concept to relate the thickness of the normal deposits to the thickness of the diffusion layer, which governs the mass transport. The force due to the nonuniform magnetic field (the field gradient force) is only effective for modifying the concentration profile in the electrolyte when there already exists a concentration gradient in the orthogonal direction. The magnetic pressure model accounts for the thickness and morphology of the direct deposits in a semiquantitative manner.

The inverse patterns can also be understood in terms of magnetic pressure acting on the electroactive solution, which has greatly enhanced effective diamagnetism due to the presence of the strongly paramagnetic rare-earth background. However, in this case the growth process cannot be accounted for by onedimensional diffusion perpendicular to the electrode surface. Radial diffusion and flow is predominant, and growth beneath the magnet is inhibited by the concentration of $\mathrm{Dy}^{3+}$ ions there.

The magnetically induced textures in the deposits are poorly understood. They can be associated to some extent with the 
growth rate, but there are magnetic field effects on nucleation in the first seconds, which merit detailed study and explanation.

While we have focused here on dot arrays, which have the advantages that the patterns can be easily related to the form of the array, other shapes of electrodeposits such as lines, can be produced, which mirror the distribution of the component of magnetic field normal to the cation surface. ${ }^{53}$

\section{ACKNOWLEDGMENTS}

This work was supported by Science Foundation Ireland as part of the RFP and NISE projects, Contract No. 09/RFP/PHY2379 and 10/IN1/I3002. We thank Plamen Stamenov for much helpful criticism, and Karl Ackland for the FIB cross sections.
*Present address: Leibniz Institute for Solid State and Materials Research, IFW Dresden, P.O. Box 270116, D-01171 Dresden, Germany; pdunne2@tcd.ie

${ }^{1}$ R. Aogaki, K. Fueki, and T. Mukaibo, Denki Kagaku 43, 509 (1975).

${ }^{2}$ R. Aogaki, K. Fueki, and T. Mukaibo, Denki Kagaku 44, 89 (1976).

${ }^{3}$ G. Hinds, F. E. Spada, J. M. D. Coey, T. R. Ní Mhíocháin, and M. E. G. Lyons, J. Phys. Chem. B 105, 9487 (2001).

${ }^{4}$ O. Devos, A. Olivier, J. P. Chopart, O. Aaboubi, and G. Maurin, J. Electrochem. Soc. 145, 401 (1998).

${ }^{5}$ M. Uhlemann, A. Krause, J. P. Chopart, and A. Gebert, J. Electrochem. Soc. 152, C817 (2005).

${ }^{6}$ M. Uhlemann, H. Schlorb, K. Msellak, and J.-P. Chopart, J. Electrochem. Soc. 151, C598 (2004).

${ }^{7}$ P. Fricoteaux, B. Jonvel, and J.-P. Chopart, J. Phys. Chem. B 107, 9459 (2003).

${ }^{8}$ T. Z. Fahidy, Prog. Surf. Sci. 68, 155 (2001).

${ }^{9}$ J. A. Koza, F. Karnbach, M. Uhlemann, J. McCord, C. Mickel, A. Gebert, S. Baunack, and L. Schultz, Electrochim. Acta 55, 819 (2010).

${ }^{10}$ M. Uhlemann, A. Krause, and A. Gebert, J. Electroanal. Chem. 577, 19 (2005).

${ }^{11}$ D. Fernández, Z. Diao, P. Dunne, and J. M. D. Coey, Electrochim. Acta 55, 8664 (2010).

${ }^{12}$ Z. H. Gu, J. Chen, A. Olivier, and T. Z. Fahidy, J. Electrochem. Soc. 140, 408 (1993).

${ }^{13}$ C. Wang, S. Chen, and H. Ma, J. Electrochem. Soc. 145, 2214 (1998).

${ }^{14}$ A. Bund and H. H. Kuehnlein, J. Phys. Chem. B 109, 19845 (2005).

${ }^{15}$ A. Dass, J. A. Counsil, X. Gao, and N. Leventis, J. Phys. Chem. B 109, 11065 (2005).

${ }^{16}$ Y. C. Tang and A. J. Davenport, J. Electrochem. Soc. 154, C362 (2007).

${ }^{17}$ M. Asanuma, A. Yamada, and R. Aogaki, Jpn. J. Appl. Phys. 44, 5137 (2005).

${ }^{18}$ F. M. F. Rhen and J. M. D. Coey, J. Phys. Chem. C 111, 3412 (2007)

${ }^{19}$ M. D. Pullins, K. M. Grant, and H. S. White, J. Phys. Chem. B 105, 8989 (2001).

${ }^{20}$ N. Leventis and X. Gao, Anal. Chem. 73, 3981 (2001).

${ }^{21}$ R. Sueptitz, K. Tschulik, M. Uhlemann, A. Gebert, and L. Schultz, Electrochim. Acta 55, 5200 (2010).

${ }^{22}$ K. Tschulik, J. A. Koza, M. Uhlemann, A. Gebert, and L. Schultz, Electrochem. Commun. 11, 2241 (2009).

${ }^{23}$ K. Tschulik, R. Sueptitz, J. Koza, M. Uhlemann, G. Mutschke, T. Weier, A. Gebert, and L. Schultz, Electrochim. Acta 56, 297 (2010).

${ }^{24}$ P. Dunne, L. Mazza, and J. M. D. Coey, Phys. Rev. Lett. 107, 024501 (2011).
${ }^{25}$ K. Tschulik, X. Yang, G. Mutschke, M. Uhlemann, K. Eckert, R. Sueptitz, L. Schultz, and A. Gebert, Electrochem. Commun. 13, 946 (2011).

${ }^{26}$ A. J. Bard and L. R. Faulkner, Electrochemical Methods: Fundamentals and Applications, 2nd ed. (Wiley, New York, 2000).

${ }^{27}$ C. Akilan, G. Hefter, N. Rohman, and R. Buchner, J. Phys. Chem. B 110, 14961 (2006).

${ }^{28}$ J. M. D. Coey, Magnetism and Magnetic Materials (Cambridge University Press, Cambridge, 2010).

${ }^{29}$ G. Hinds, J. M. D. Coey, and M. E. G. Lyons, Electrochem. Commun. 3, 215 (2001).

${ }^{30} \mathrm{G}$. Mutschke, K. Tschulik, T. Weier, M. Uhlemann, A. Bund, and J. Fröhlich, Electrochim. Acta 55, 9060 (2010).

${ }^{31}$ C. M. A. Brett and A. M. Oliveira-Brett, Electrochemistry: Principles, Methods, and Applications (Oxford University Press, Oxford, 2000).

${ }^{32}$ J. M. D. Coey, F. M. F. Rhen, P. Dunne, and S. McMurry, J. Solid State Electrochem. 11, 711 (2007).

${ }^{33}$ P. A. Dunne, J. Hilton, and J. M. D. Coey, J. Magn. Magn. Mater. 316, 273 (2007).

${ }^{34}$ K. P. Musselman, T. Gershon, L. Schmidt-Mende, and J. L. MacManus-Driscoll, Electrochim. Acta 56, 3758 (2011).

${ }^{35}$ R. F. Large and A. Timnick, Anal. Chem. 36, 1258 (1964).

${ }^{36}$ R. Aogaki, E. Ito, and M. Ogata, J. Solid State Electrochem. 11, 757 (2007).

${ }^{37}$ J. M. D. Coey, R. Aogaki, F. Byrne, and P. Stamenov, Proc. Natl. Acad. Sci. USA 106, 8811 (2009).

${ }^{38}$ K. Mogi, T. Sakihama, N. Hirota, and K. Kitazawa, J. Appl. Phys. 85, 5714 (1999).

${ }^{39}$ N. Hirota, Y. Ikezoe, H. Uetake, T. Kaihatsu, T. Takayama, and K. Kitazawa, RIKEN Rev. 44, 159 (2002).

${ }^{40}$ H. Sugawara, N. Hirota, T. Homma, M. Ohta, K. Kitazawa, H. Yokoi, Y. Kakudate, S. Fujiwara, M. Kawamura, S. Ueno, and M. Iwasaka, J. Appl. Phys. 79, 4721 (1996).

${ }^{41}$ R. E. Rosensweig, Ferrohydrodynamics (Courier Dover Publications, New York, 1997).

${ }^{42} \mathrm{P}$. A. Davidson, An Introduction to Magnetohydrodynamics (Cambridge University Press, Cambridge, 2001).

${ }^{43}$ T. R. Ní Mhíocháin and J. M. D. Coey, Phys. Rev. E 69, 061404 (2004).

${ }^{44}$ A.-L. Daltin, F. Bohr, and J.-P. Chopart, Electrochim. Acta 54, 5813 (2009).

${ }^{45}$ T. Z. Fahidy, in Modern Aspects of Electrochemistry, edited by B. E. Conway, J. O. Bockris, and R. E. White (Springer, New York, 2002), pp. 333-354.

${ }^{46}$ J. Koza, M. Uhlemann, A. Gebert, and L. Schultz, J. Solid State Electrochem. 12, 181 (2008). 
${ }^{47}$ R. Aogaki, R. Morimoto, and M. Asanuma, J. Magn. Magn. Mater. 322, 1664 (2010).

${ }^{48}$ W. Shao and G. Zangari, J. Phys. Chem. C 113, 10097 (2009).

${ }^{49}$ Y. Ikezoe, N. Hirota, J. Nakagawa, and K. Kitazawa, Nature 393, 749 (1998).
${ }^{50}$ E. Beaugnon and R. Tournier, Nature 349, 470 (1991).

${ }^{51}$ M. V. Berry and A. K. Geim, Eur. J. Phys 18, 307 (1997).

${ }^{52}$ M. Motokawa, M. Hamai, T. Sato, I. Mogi, S. Awaji, K. Watanabe, N. Kitamura, and M. Makihara, Physica B 294, 729 (2001).

${ }^{53}$ P. Dunne, R. Soucaille, K. Ackland, and J. M. D. Coey, J. Appl. Phys. 111, 07B915 (2012). 